\title{
SP12RTS: a degree-12 model of shear- and compressional-wave velocity for Earth's mantle
}

\author{
P. Koelemeijer, ${ }^{1,2}$ J. Ritsema, ${ }^{3}$ A. Deuss ${ }^{4}$ and H.-J. van Heijst ${ }^{5}$ \\ ${ }^{1}$ Bullard Laboratories, Department of Earth Sciences, University of Cambridge, Cambridge CB3 0EZ, United Kingdom. E-mail: pjkoelemeijer@cantab.net \\ ${ }^{2}$ Institute of Geophysics, Department of Earth Sciences, ETH Zürich, 8092 Zurich, Switzerland \\ ${ }^{3}$ Department of Earth and Environmental Sciences, University of Michigan, Ann Arbor, MI 48109, USA \\ ${ }^{4}$ Department of Earth Sciences, Utrecht University, 3508 TC Utrecht, The Netherlands \\ ${ }^{5}$ Shell International Exploration and Production, 2501 CR Den Haag, The Netherlands
}

Accepted 2015 November 2. Received 2015 October 22; in original form 2015 August 7

\begin{abstract}
S UMMAR Y
We present the new model SP12RTS of isotropic shear-wave $\left(V_{S}\right)$ and compressional-wave $\left(V_{P}\right)$ velocity variations in the Earth's mantle. SP12RTS is derived using the same methods as employed in the construction of the shear-wave velocity models S20RTS and S40RTS, and the same data types. SP12RTS includes additional traveltime measurements of $P$-waves and new splitting measurements: 33 normal modes with sensitivity to the compressional-wave velocity and 9 Stoneley modes with sensitivity primarily to the lowermost mantle. Contrary to S20RTS and S40RTS, variations in $V_{S}$ and $V_{P}$ are determined without invoking scaling relationships. Lateral velocity variations in SP12RTS are parametrised using spherical harmonics up to degree 12, to focus on long-wavelength features of $V_{S}$ and $V_{P}$ and their ratio $R$. Large-lowvelocity provinces (LLVPs) are observed for both $V_{S}$ and $V_{P}$. SP12RTS also features an increase of $R$ up to $2500 \mathrm{~km}$ depth, followed by a decrease towards the core-mantle boundary. A negative correlation between the shear-wave and bulk-sound velocity variations is observed for both the LLVPs and the surrounding mantle. These characteristics can be explained by the presence of post-perovskite or large-scale chemical heterogeneity in the lower mantle.
\end{abstract}

Key words: Composition of the mantle; Body waves; Surface waves and free oscillations; Seismic tomography.

\section{INTRODUCTION}

The core-mantle boundary (CMB) region is a dynamic region within the Earth. Lateral variations in velocity and density within $\mathrm{D}^{\prime \prime}$, the lowermost few hundred kilometres of the mantle, represent variations in temperature and chemical composition (see Garnero \& McNamara (2008) and Lay \& Garnero (2011) for reviews). Since the study of Dziewonski et al. (1977), numerous maps have been published of shear-wave velocity variations $\mathrm{d} \ln V_{S}\left(=\mathrm{d} V_{S} / V_{S}\right)($ e.g. Ritsema et al. 1999; Gu et al. 2001; Panning \& Romanowicz 2006; Kustowski et al. 2008; Ritsema et al. 2011; French et al. 2013; Auer et al. 2014; Chang et al. 2015), compressional-wave velocity variations $\operatorname{d} \ln V_{P}$ (e.g. Boschi \& Dziewonski 2000; Li et al. 2008; Soldati et al. 2012; Young et al. 2013), joint shear-wave and bulk-sound velocity variations $\operatorname{dln} V_{C}$ (e.g. Su \& Dziewonski 1997; Masters et al. 2000; Antolik et al. 2003; Houser et al. 2008) and density variations dln $\rho$ (e.g. Ishii \& Tromp 1999, 2001; Trampert et al. 2004; Mosca et al. 2012).

The clustering analysis of Lekic et al. (2012) illustrates that a consistent picture of the shear-wave velocity structure of the lowermost mantle is now emerging. Two antipodal, large-low-shearvelocity provinces (LLSVPs) cover almost half of the core surface and extend up to $1000 \mathrm{~km}$ above the CMB. The LLSVPs and the surrounding 'ring around the Pacific' of higher-than-average velocities determine the long-wavelength structure of the lower mantle, with high amplitudes at spherical harmonic degrees two and three. Nevertheless, it remains uncertain whether the LLSVPs are large-scale thermal anomalies, plume clusters distorted by limited tomographic resolution (e.g. Davies \& Davies 2009; Schuberth et al. 2009, 2012; Davies et al. 2012), or stable, chemically distinct, dense piles (e.g. Davaille 1999; McNamara \& Zhong 2005; Deschamps et al. 2007; Deschamps \& Tackley 2009; McNamara et al. 2010; Nakagawa et al. 2012), and whether they actively influence mantle dynamics (e.g. Dziewonski et al. 2010) or passively migrate due to impinging subducting slabs (e.g. Bower et al. 2013). The presence of large-scale chemical heterogeneity also has important implications for the nature of mantle convection, Earth's heat budget and the evolution of the core (e.g. Lay et al. 2008; Nakagawa \& Tackley 2014).

Models of the density variations in the lower mantle will directly constrain the buoyancy of the LLSVPs and answer these questions. Unfortunately, density variations are not easily constrained from the available seismic data. Normal-mode models suggest that the LLSVPs have a relatively high density (Ishii \& Tromp 1999, 2001; 
Trampert et al. 2004), but the resolvability of density variations has been questioned (Resovsky \& Ritzwoller 1999; Romanowicz 2001; Koelemeijer et al. 2015).

Here, we focus on shear-wave and compressional-wave velocity variations instead and, in particular, on their ratio $R=\mathrm{d} \ln V_{S} / \mathrm{d} \ln V_{P}$. Mineral physics experiments (Karato \& Karki 2001) indicate that $R<2-2.5$ in an isochemical lower mantle, which also implies that variations in shear-wave and bulk-sound velocity are positively correlated. Previous analyses have found a negative correlation between the bulk-sound and shear-wave velocity variations, either throughout the entire lower mantle (e.g. Su \& Dziewonski 1997) or limited to deeper depths (e.g. Masters et al. 2000). Most studies find that $R$ is $\sim 1.5$ at the top of the lower mantle and as high as 3.5 at the CMB (Su \& Dziewonski 1997; Masters et al. 2000; Ritsema \& van Heijst 2002; Antolik et al. 2003; Della Mora et al. 2011). The high value of $R$ may indicate that the lowermost mantle has a different composition (e.g. Su \& Dziewonski 1997; Masters et al. 2000; Houser et al. 2008) or the presence of the post-perovskite (pPv) phase in the lower mantle (e.g. Iitaka et al. 2004; Murakami et al. 2004; Oganov \& Ono 2004; Tsuchiya et al. 2004; Wookey et al. 2005; Davies et al. 2012). Whether the stability field of $\mathrm{pPv}$ extends high enough above the CMB remains uncertain (see review by Cobden et al. (2015)). Furthermore, it has been suggested that estimates of $R$ and the correlation between $\operatorname{d} \ln V_{S}$ and $\operatorname{d} \ln V_{C}$ could be influenced by finite-frequency effects on body-wave traveltimes (Malcolm \& Trampert 2011; Schuberth et al. 2012), although high values of $R$ and a negative correlation between $\operatorname{dn} \ln V_{S}$ and $\operatorname{d} \ln V_{C}$ have also been observed in normal-mode derived models (Masters et al. 2000; Trampert et al. 2004), which correctly take finite-frequency effects into account.

In this paper, we present the result of a new joint inversion of traveltime, surface-wave and normal-mode data for $V_{S}$ and $V_{P}$ structure in the lower mantle and $R$ in particular. In contrast to body waves, normal modes automatically provide uniform sampling of both $V_{S}$ and $V_{P}$ in the lowermost mantle, which is essential to accurately constrain their relative amplitudes. Furthermore, normalmode measurements are obtained by a full waveform fit to seismic spectra and hence automatically include finite-frequency effects. Even more importantly, we include new measurements of modes sensitive to $V_{P}$ structure in our normal-mode data set, based on large megathrust events and several large continental earthquakes from the past four decades (Deuss et al. 2013). We also add new measurements of CMB Stoneley modes that are primarily sensitive to the lowermost mantle and have never been employed in inversions for mantle structure (Koelemeijer et al. 2013). In contrast, until recently the normal-mode data sets employed in tomographic studies were primarily sensitive to the shear-wave velocity structure of the mantle and did not contain Stoneley modes (e.g. Ritsema et al. 2011; Mosca et al. 2012).

We use the inversion framework of the shear-wave velocity models S20RTS (Ritsema et al. 1999, RHW99 from hereon), S20RTSb (Ritsema et al. 2004, RHW04 from hereon) and S40RTS (Ritsema et al. 2011, RDHW11 from hereon). Rather than assuming a uniform (e.g. RHW99, RHW04) or depth-dependent (RDHW11) scaling between $\operatorname{d} \ln V_{S}$ and $\operatorname{d} \ln V_{P}$, we invert for both shear- and compressional-wave velocity variations independently without invoking any scaling. Here, we focus on presenting the new model SP12RTS, showing the influence of CMB Stoneley mode data and comparing the obtained values of $R$ to previous studies. Interpretation of its characteristics will be done in combination with mineral physics and geodynamic modelling and is hence left to forthcoming studies.

\section{DATA}

Akin to RDHW11, we combine normal-mode splitting function coefficients, Rayleigh wave phase-velocity measurements and teleseismic body-wave traveltimes to optimise data coverage. We include $P$-wave traveltimes and expand the normal-mode splitting measurements up to $10 \mathrm{mHz}$. Our focus on the long-wavelength structure greatly reduces the number of parameters in our inversion and we therefore reprocess the body-wave and surface-wave data sets with stricter criteria.

\subsection{Body-wave traveltimes}

The body-wave data set includes nearly 700000 measurements of the delay times of teleseismic $P$ - and $S$-waves from 4250 events between 1984 and 2008 with a body-wave magnitude larger than 5.9. We measure the delay times by cross-correlating low-pass ( $T>16 \mathrm{~s}$ ) filtered seismograms and normal-mode synthetics (Ritsema \& van Heijst 2002). The noise level of the data, waveform fits and wave amplitude discrepancies are used to quantify the quality of the measurements. These body-wave data are particularly important to provide coverage in the lower mantle and constraints on odd degree structure.

The data set of RDHW11 is supplemented with measurements of the delay times of $P, P_{\mathrm{diff}}, P P, P P P, P K P_{\mathrm{ab}}$ and $P K P_{\mathrm{df}}$ and their accompanying depth phases. The teleseismic body waves provide global coverage of the lower mantle (Fig. 1). The $P$ phases turn in the lower third (2000-2800 km depth) of the mantle, whereas $P P(800$ $2000 \mathrm{~km})$ and $P P P(800-1400 \mathrm{~km})$ turn in the upper half of the lower mantle. $P_{\text {diff }}$ propagates horizontally through $\mathrm{D}^{\prime \prime}$ whereas $P K P_{\mathrm{df}}$ propagates almost vertically through the Earth's mantle. In total, we include $297134 S$-wave phases and $390455 P$-wave phases listed in Table 1. Each traveltime measurement is weighted equally in the inversion. We refer the reader to Ritsema \& van Heijst (2002) for details on the traveltime measurement procedure and to RDHW11 for a discussion of the $S$-wave phases.

\subsection{Rayleigh wave phase velocities}

Surface-wave dispersion of the fundamental and first four overtone branches is measured using the mode-branch-stripping technique of Van Heijst \& Woodhouse (1997, 1999) for all earthquakes of the Global CMT catalogue up to 2008. The overtone measurements comprise about a third of our phase-velocity data. The updated data set contains about four million phase-velocity measurements, derived from both vertical and horizontal component long-period seismograms (Table 2). These surface-wave data are particularly important for constraining the upper mantle and transition zone to prevent leakage of structure into the lowermost mantle. The reader is referred to Van Heijst \& Woodhouse $(1997,1999)$ for details on the overtone measurements and phase-velocity maps, to RHW04 for details on the inversion procedure and to RDHW11 for details regarding the expansion of the data set until 2008.

\subsection{Splitting functions}

Given our interest in the long-wavelength structure of the lower mantle, long-period normal-mode data are particularly important. Direct inversion of modal spectra is a very non-linear procedure. Therefore, we use the splitting function approach, which serves as a very convenient intermediate stage for modelling Earth's structure (Li et al. 1991b). Splitting functions are measured by iterative 
(a) $\mathbf{P}$

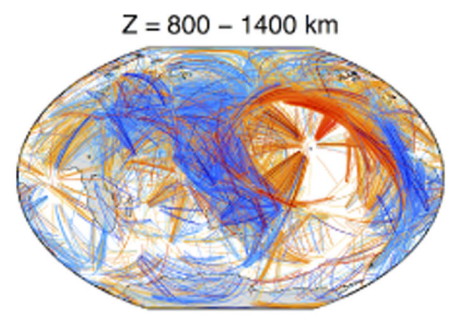

$Z=1400-2000 \mathrm{~km}$

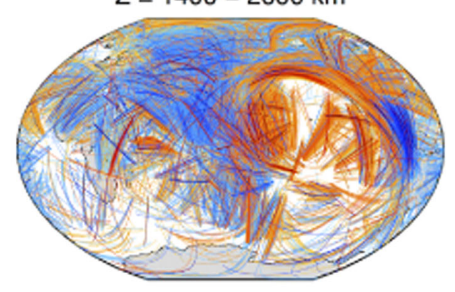

$Z=2000-2800 \mathrm{~km}$

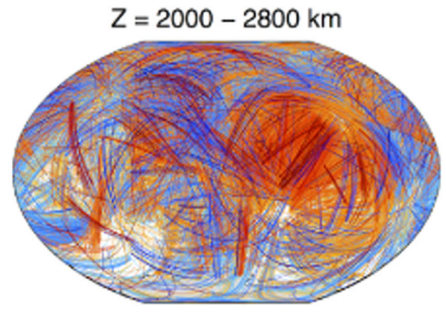

(b) PP
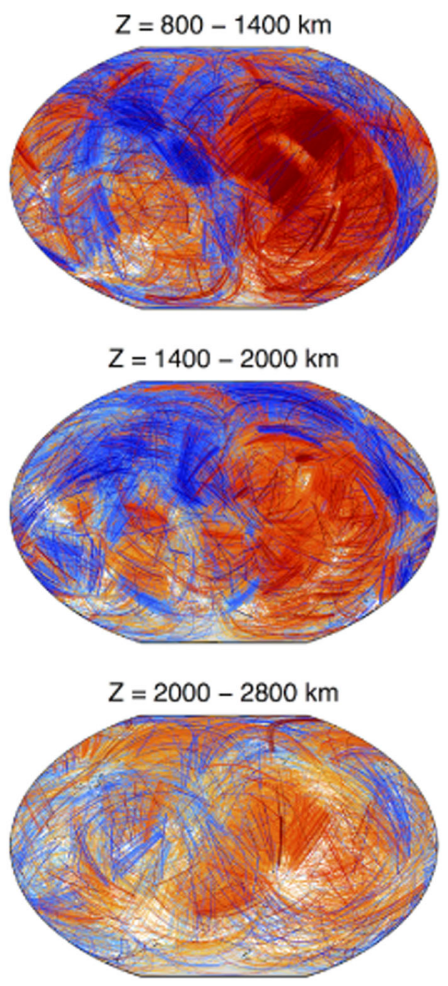

(c) PPP

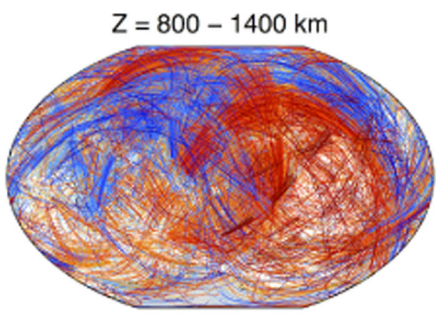

(d) $P_{\text {diff }}$

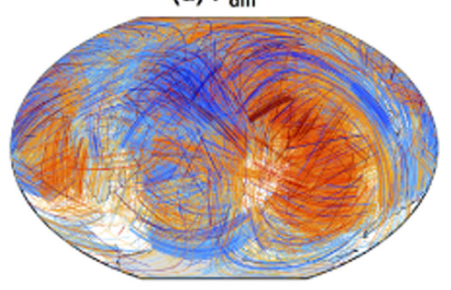

(e) PKP $_{d f}$

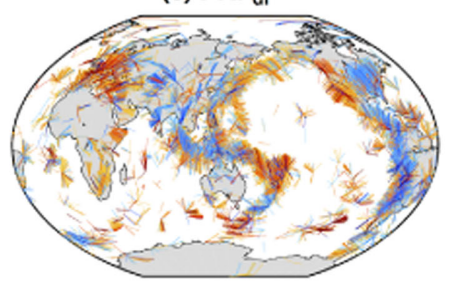

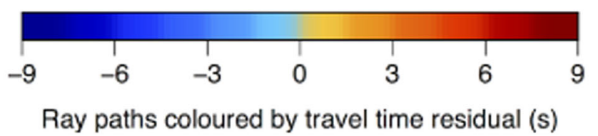

Figure 1. Ray path segments of (a) the direct $P$, (b) the surface-reflected $P P$, (c) the twice surface-reflected $P P P$, (d) the core-diffracted $P_{\text {diff }}$ and (e) the inner core $P K P_{\text {df }}$ phase. The segments in (a)-(c) are limited to depth intervals of (top) $800-1400 \mathrm{~km}$, (middle) 1400-2000 km and (bottom) $2000-2800 \mathrm{~km}$ where $P, P P$ and $P P P$ turn. In (d) the diffracted paths and in (e) the entire mantle paths are plotted. The ray path segments are coloured by their traveltime residuals, which are corrected for ellipticity, earthquake mislocation and crustal structure (Ritsema \& van Heijst 2002).

Table 1. Included body-wave phases, total number of paths for each phase and their associated L2 data-normalized model misfit.

\begin{tabular}{lrllrl}
\hline Phase & Paths & Misfit & Phase & Paths & Misfit \\
\hline$S, S_{\text {diff }}$ & 135163 & 0.352 & $P, P_{\text {diff }}$ & 207631 & 0.600 \\
$S S$ & 75912 & 0.269 & $P P$ & 127241 & 0.391 \\
$S S S$ & 11902 & 0.293 & $P P P$ & 7307 & 0.775 \\
$S K S$ & 23838 & 0.632 & $P K P_{\text {df }}$ & 15687 & 0.878 \\
$S K K S$ & 6109 & 0.662 & $P K P_{\mathrm{ab}}$ & 664 & 0.859 \\
$S c S$ & 5545 & 0.419 & $p P$ & 22841 & 0.587 \\
$S c S_{2}$ & 6896 & 0.277 & $p P P$ & 7941 & 0.339 \\
$S c S_{3}$ & 3736 & 0.150 & $p P P P$ & 1143 & 0.578 \\
$s S, s S_{\text {diff }}$ & 15011 & 0.279 & & & \\
$S S S$ & 6146 & 0.268 & & & \\
$S S S S$ & 1241 & 0.332 & & & \\
$S S K S$ & 1556 & 0.474 & & & \\
$S S C S$ & 1074 & 0.385 & & & \\
$S S c S_{2}$ & 1828 & 0.138 & & & \\
$S S c S_{3}$ & 1177 & 0.148 & & & \\
$A l l$ & $\mathbf{2 9 7} \mathbf{1 3 4}$ & $\mathbf{0 . 3 4 7 5}$ & All & $\mathbf{3 9 0 4 5 5}$ & $\mathbf{0 . 5 4 0 6}$
\end{tabular}

fitting of modal spectra for 93 earthquakes with $M_{w}>7.4$ between 1976 and 2011 using the method of Deuss et al. (2013), similar to Li et al. (1991b). We only use even-degree self-coupled splitting function coefficients in our inversions, although for some modes cross-coupling was included in the splitting function measurement itself.

The normal-mode data set of RDHW11 is expanded up to frequencies of $10 \mathrm{mHz}$ using splitting function measurements of Deuss et al. (2013), including 33 new modes sensitive to $V_{P}$ structure (Fig. 2a). We exclude modes with sensitivity to the inner core as these are strongly split due to inner core anisotropy (Woodhouse et al. 1986). We combine these measurements with nine new CMB Stoneley mode splitting function measurements of Koelemeijer et al. (2013) that are uniquely sensitive to the $\mathrm{CMB}$ region (Fig. 2b). Other new splitting function measurements of higher order fundamental modes (Koelemeijer 2014) are also incorporated.

In total, we include 6970 splitting function coefficients for 143 normal modes (Table 3 ). This is a threefold increase compared with RDHW11 (with 49 modes and 2903 coefficients), with significant higher sensitivity to $V_{P}$ and lowermost mantle structure. The number of mode coefficients may seem small compared to the body-wave and surface-wave data sets but each splitting function is obtained from the inversion of typically 500-2500 spectra and more than 100000 spectra in total were used to measure the coefficients (Deuss et al. 2013). Relative weights are applied to each mode using the uncertainty estimates in the splitting functions which were determined from the maximum range in cross-validation runs (Deuss 
Table 2. Rayleigh wave phase-velocity measurements, total number of paths for each measurement and their L2 data-normalized model misfit at $T=78 \mathrm{~s}$.

\begin{tabular}{lrrrrrr}
\hline Branch & $\begin{array}{c}\text { Minor arcs } \\
\text { Period (s) }\end{array}$ & Paths & Misfit & $\begin{array}{r}\text { Major arcs } \\
\text { Period (s) }\end{array}$ & Paths & Misfit \\
\hline Fundamental mode & $321-40$ & 917675 & 0.339 & $274-34$ & 968302 & 0.190 \\
1st overtone & $274-56$ & 96962 & 0.180 & $202-88$ & 292850 & 0.251 \\
2nd overtone & $114-51$ & 47728 & 0.232 & $202-69$ & 656066 & 0.209 \\
3rd overtone & $100-43$ & 35113 & 0.481 & $114-62$ & 437138 & 0.563 \\
4th overtone & $69-47$ & 15428 & 0.353 & $88-51$ & 541865 & 0.508 \\
All & $\mathbf{3 2 1}-\mathbf{4 0}$ & $\mathbf{1 1 1 2 9 0 6}$ & $\mathbf{0 . 3 1 8 4}$ & $\mathbf{2 7 4 - \mathbf { 3 4 }}$ & $\mathbf{2 8 9 6 \mathbf { 2 2 1 }}$ & $\mathbf{0 . 4 5 8 3}$ \\
\hline
\end{tabular}

(a) P-wave sensitive mode ${ }_{9} S_{13}, s=8$

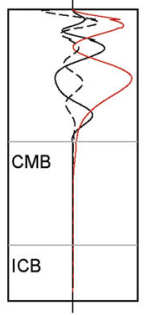

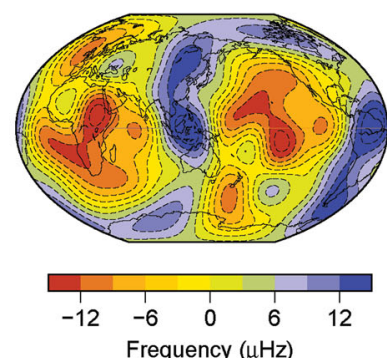

(b) CMB Stoneley mode ${ }_{2} \mathrm{~S}_{16}, \mathrm{~s}=6$

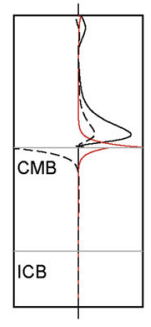

3. Splitting function measurements and their associated L2 datanormalized model misfit.

\begin{tabular}{|c|c|c|c|}
\hline Branch & Modes & Coefficients & Misfit \\
\hline 0 & ${ }_{0} S_{3-0} S_{9},{ }_{0} S_{12-0} S_{30}$ & 1963 & 0.081 \\
\hline 1 & ${ }_{1} S_{3-1} S_{16}$ & 639 & 0.067 \\
\hline 2 & ${ }_{2} S_{4-2} S_{17}, 2 S_{25}$ & 798 & 0.079 \\
\hline 3 & ${ }_{3} S_{6-3} S_{9,}{ }_{3} S_{25-3} S_{26}$ & 243 & 0.086 \\
\hline 4 & ${ }_{4} S_{1-4} S_{5}$ & 96 & 0.115 \\
\hline 5 & ${ }_{5} S_{3-5} S_{8},{ }_{5} S_{11-5} S_{12},{ }_{5} S_{14-5} S_{17}$ & 611 & 0.065 \\
\hline 6 & ${ }_{6} S_{9-6} S_{10,} S_{15},{ }_{6} S_{18}$ & 205 & 0.123 \\
\hline 7 & ${ }_{7} S_{5-7} S_{9}$ & 174 & 0.101 \\
\hline 8 & ${ }_{8} S_{6-8} S_{7},{ }_{8} S_{10}$ & 135 & 0.065 \\
\hline 9 & ${ }_{9} S_{6},{ }_{9} S_{8},{ }_{9} S_{10-9} S_{15}$ & 330 & 0.068 \\
\hline 10 & ${ }_{10} S_{10},{ }_{10} S_{17-10} S_{21}$ & 206 & 0.043 \\
\hline \multirow[t]{2}{*}{11} & ${ }_{11} S_{9-11} S_{10},{ }_{11} S_{12},{ }_{11} S_{14}$ & & \\
\hline & ${ }_{11} S_{23-11} S_{25}$ & 289 & 0.151 \\
\hline 12 & ${ }_{12} S_{6-12} S_{8},{ }_{12} S_{11-12} S_{17}$ & 415 & 0.124 \\
\hline 13 & ${ }_{13} S_{15-13} S_{16},{ }_{13} S_{18-13} S_{20}$ & 229 & 0.063 \\
\hline 14 & ${ }_{14} S_{7-14} S_{9},{ }_{14} S_{13-14} S_{14}$ & 229 & 0.097 \\
\hline 15 & ${ }_{15} S_{12},{ }_{15} S_{15-15} S_{16}$ & 139 & 0.081 \\
\hline 16 & ${ }_{16} S_{10-16} S_{11},{ }_{16} S_{14}$ & 84 & 0.092 \\
\hline 17 & ${ }_{17} S_{12-17} S_{15}$ & 112 & 0.101 \\
\hline 19 & ${ }_{19} S_{10-19} S_{11}$ & 73 & 0.100 \\
\hline All & & 6970 & 0.0834 \\
\hline
\end{tabular}

\subsection{Theoretical simplifications}

The resolved shear-wave velocity structure obtained using either ray theory or finite-frequency theory is essentially the same for the long-wavelength (i.e. degree 12) parametrization of SP12RTS, as long as the regularisation in the inversion is adjusted (Ritsema et al. 2011). Our long-wavelength parametrization ensures that the shortest wavelengths of our model $(\sim 1000 \mathrm{~km})$ are larger than the Fresnel zones of the dominant body-wave phases. Although it remains questionable whether ray theory suffices for determining the detailed characteristics of $R$ in the $\mathrm{D}^{\prime \prime}$ region, the overall structure of $R$ with depth in the mantle is resolvable with ray theory and readily observed in traveltime data (Ritsema \& van Heijst 2002). Therefore, in line with RHW99, RHW04 and RDHW11, we relate traveltime anomalies, phase-velocity anomalies and splitting functions to velocity heterogeneity by assuming that (i) the traveltime is affected by velocity heterogeneity along the geometric ray, (ii) the Rayleigh wave is affected by structure in the plane of propagation and (iii) splitting functions can be mapped into velocity heterogeneity using depth-dependent sensitivity kernels. This enables us to analyse a large data set and experiment with inversion parameters at moderate computational cost. Model data fits are assessed using a data-normalized L2 norm for each body-wave phase, surface-wave (in different period ranges) and normal-mode branch. For more information regarding the theoretical simplifications, we refer the reader to RHW04. 


\subsection{Parametrization}

We use the same three-point clamped cubic spline functions as RDHW11 (see Ritsema et al. 2004, their fig. 4) to parametrise velocity variations with depth. Lateral variations in shear-wave velocity $\delta V_{S}$ and compressional-wave velocity $\delta V_{P}$ are parametrised using spherical harmonic basis functions, up to angular degree $s=12$ (primarily limited by the available normal-mode data), giving:

$\delta V_{S}(\mathbf{x})=\sum_{k=1}^{K} \sum_{s=0}^{12} \sum_{t=-s}^{s} S_{s t}^{k} z^{k}(r) Y_{s}^{t}(\theta, \phi)$,

and

$\delta V_{P}(\mathbf{x})=\sum_{k=1}^{K} \sum_{s=0}^{12} \sum_{t=-s}^{s} P_{s t}^{k} z^{k}(r) Y_{s}^{t}(\theta, \phi)$,

where $S_{s t}^{k}$ and $P_{s t}^{k}$ represent coefficients describing the velocity variations, $z^{k}$ represent the splines as a function of depth, $K$ is the maximum number of splines (e.g. 21) and $Y_{s}^{t}$ are fully normalized spherical harmonics (Edmonds 1960). The parametrization up to $s=12$ accommodates structures with a half wavelength of $\sim 1600 \mathrm{~km}$ at the surface and $\sim 900 \mathrm{~km}$ at the CMB.

\subsection{Crustal corrections}

Since crustal velocity variations cannot be resolved by our data, we subtract the contributions of the crust using model CRUST2.0 (Bassin et al. 2000). Similar to RDH11, we correct the Rayleigh wave phase-velocity and normal-mode splitting function data for crustal thickness, surface topography and sea level height. Traveltime corrections are determined using normal-mode synthetics for 1-D profiles of CRUST2.0. Such 'crustal corrections', would affect estimates of anisotropy in the upper mantle (Ferreira et al. 2010; Chang et al. 2015), but isotropic deep mantle $V_{S}$ models are robust with respect to changes in crustal corrections (Panning et al. 2010). Whether significant $V_{P}$ anisotropy is present in the lowermost mantle and whether isotropic lower mantle $V_{P}$ models are severely affected by crustal corrections is still unknown. The reader is referred to RDHW11 for more details regarding these crustal corrections.

\subsection{Inversion procedure}

Our inversion procedure is identical to that of RDHW11, except that the model vector $\mathbf{m}$ now has two elements:

$\mathbf{m}=\left(\begin{array}{l}\mathbf{S} \\ \mathbf{P}\end{array}\right)$

where $\mathbf{S}$ and $\mathbf{P}$ describe the shear-wave velocity variations $\delta V_{S}(\mathbf{x})$ and compressional-wave velocity variations $\delta V_{P}(\mathbf{x})$ of (1) and (2), relative to the reference model PREM.

The relationships between $\mathbf{m}$ and the data vector $\mathbf{d}$ containing the traveltime, phase-velocity and splitting function measurements can be written in a linear way:

$\mathbf{d}=\mathbf{G m}$,

where $\mathbf{G}$ is the matrix containing all partial derivatives.

We estimate $\mathbf{m}$ by damped least-squares inversion (Tarantola 1987; Menke 1989) to minimise:

$\Gamma(\mathbf{m})=(\mathbf{G m}-\mathbf{d})^{T}(\mathbf{G m}-\mathbf{d})+\epsilon \mathbf{m}^{T} \mathbf{m}$, where $\epsilon$ is the damping factor. We apply the same damping for $\mathbf{S}$ and $\mathbf{P}$ and $\epsilon$ is independent of depth.

The estimated solution $\mathbf{m}^{\dagger}$ to eq. (4) is:

$\mathbf{m}^{\dagger}=\mathbf{G}^{\dagger} \mathbf{d}$,

with $\mathbf{G}^{\dagger}$ the generalised inverse of $\mathbf{G}$. If $\mathbf{U} \Lambda \mathbf{U}^{T}$ is the eigenvalue decomposition of $\mathbf{G}^{T} \mathbf{G}$, then the generalised inverse is:

$\mathbf{G}^{\dagger}=\mathbf{U} \Lambda^{-1} \mathbf{U}^{T} \mathbf{G}^{T}$,

where $\Lambda^{-1}=(\Lambda+\epsilon)^{-1}$. Combining (4) and (6) yields:

$\mathbf{m}^{\dagger}=\mathbf{G}^{\dagger} \mathbf{G m}=\mathcal{R} \mathbf{m}$,

where $\mathcal{R}$ is the resolution operator that specifies how the true Earth model $\mathbf{m}$ is mapped into the tomographic model $\mathbf{m}^{\dagger}$. The spatially heterogeneous resolution is fully described by $\mathcal{R}$ and the effective number of unknowns $\mathcal{N}$ of model $\mathbf{m}^{\dagger}$ is given by the trace of $\mathcal{R}$.

Similar to RDHW11, we compute the eigenvalue decomposition of $\mathbf{G}^{T} \mathbf{G}$ to determine models $\mathbf{m}^{\dagger}$ and data misfit as a function of damping and $\mathcal{N}$ (Fig. 3). As expected, the misfit decreases with increasing $\mathcal{N}$ and decreasing $\epsilon$ as short-scale velocity structure is included. Although the misfit of the fundamental mode dispersion data continues to decrease, we choose the preferred model $\mathbf{m}^{\dagger}$ to have $\mathcal{N}=1200$ effective unknowns (indicated by the arrows in Fig. 3). For $\mathcal{N}=1200$, the splitting function and traveltime data have been optimally fit by long-wavelength structures in the lower mantle, the current region of interest. The obtained amplitudes of heterogeneity continue to increase for lower damping values (similar to RDHW11, their fig. 6), but the patterns of heterogeneity remain stable. The characteristics of SP12RTS discussed in following sections are robust across several orders of magnitude of $\epsilon$.

Fig. 4 visualizes the resolution matrix $\mathcal{R}$ for several coefficients $\mathcal{R}_{s t}$ of different spherical harmonic degree. It shows the recovered amplitude at radial spline $j$ (vertical axis) due to a unit input at radial spline $i$ (horizontal axis). Although the resolution matrix has a diagonal form, the recovered amplitude varies with spline number (i.e. with depth) and some projection of structure occurs between splines 20 and 21 in the lowermost mantle and neighbouring splines (1-6) in the upper mantle. For the upper mantle, the amplitude recovery of $\operatorname{d} \ln V_{S}$ is better than $\operatorname{d} \ln V_{P}$ since the surface-wave dispersion data have a larger sensitivity to $V_{S}$ structure. In the lower mantle, structure is primarily constrained by the traveltime and splitting function data and the resolution of $\operatorname{d} \ln V_{S}$ and $\operatorname{d} \ln V_{P}$ is comparable. Projection of $V_{P}$ structure into $V_{S}$ structure occurs (indicated by the non-zero amplitudes in the off-diagonal blocks) for spline 1 ( $25 \mathrm{~km}$ depth). $V_{S}$ structure projects into $V_{P}$ structure for splines (6-10) in the mantle transition zone and at spline 20 (2600 km depth). At these depths, the sensitivity of our data to either $V_{S}$ or $V_{P}$ is small. The vertical projection is strongest for the highest spherical harmonic degrees, especially for $\operatorname{d} \ln V_{P}$. Therefore, we limit our interpretation to the LLSVPs and the surrounding regions (SRs) that are primarily described by spherical harmonic degrees up to $s=8$.

Data misfit and model resolution depend on the chosen relative weights of the data sets and the damping value $\epsilon$ used in the inversion. To ensure meaningful comparisons, we only compare models with the same number of resolved parameters, that is, $N=1200$, in the following sections.

\subsection{Data sensitivity and weighting}

To understand the contribution of each data set, we visualise the data sensitivity to mantle structure within our chosen parametrization. 
(a) Dispersion data

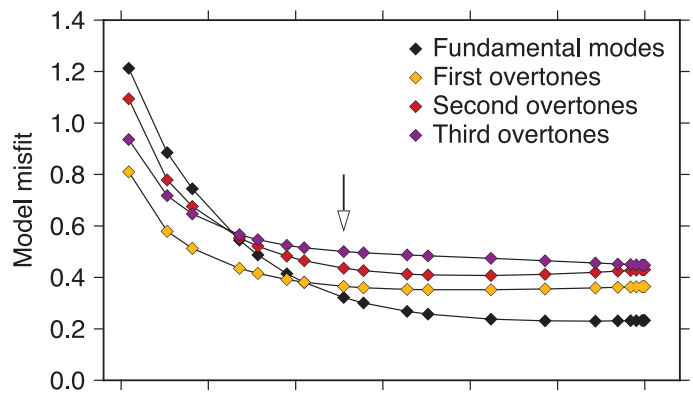

(b) Splitting data

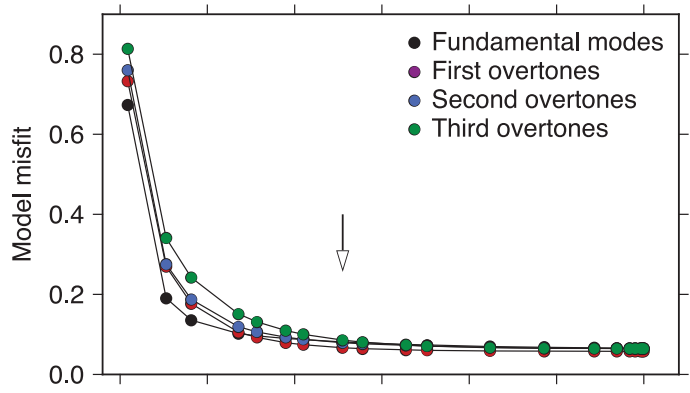

(c) Traveltime data

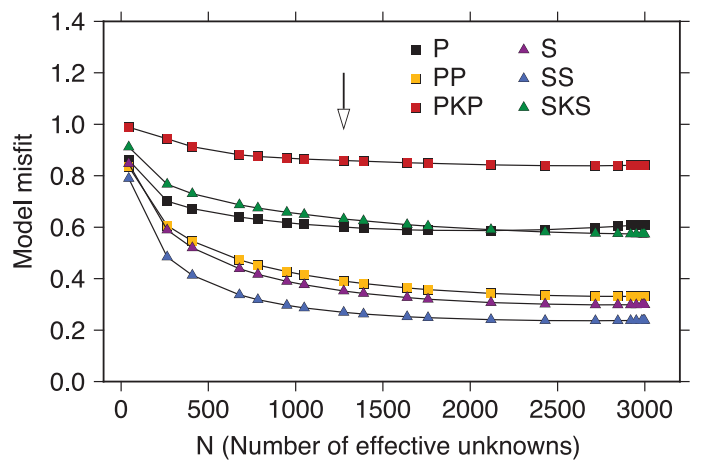

Figure 3. Data-normalized L2 misfit as a function of $\mathcal{N}$ for subsets of the data showing (a) Rayleigh wave phase-velocity measurements of the fundamental, first-, second- and third-order overtone branches (summed over all periods available), (b) splitting function measurements for the same branches and (c) traveltime delays of $P, P P, P K P, S, S S$ and $S K S$. The arrow indicates our choice of damping parameter corresponding to about 1200 unknowns. The decrease in misfit is relatively small for $\mathcal{N}>1200$, except for the fundamental surface waves that mainly have sensitivity to the uppermost mantle. Misfit curves shown here correspond to those of the final SP12RTS model, which is presented in Section 4.

The sensitivity to the spline function at each radial knot $k$ is directly related to the corresponding elements of the $\mathbf{G}^{T} \mathbf{G}$ matrix. Following Gu et al. (2001), we define for each $j$ th data set a simple horizontal average for the $k$ th spline:

$\widetilde{\left(\mathbf{G}^{T} \mathbf{G}\right)_{k}^{j}}=\widetilde{\mathbf{g}}_{k}^{j}=\sqrt{\frac{1}{4 \pi} \sum_{s t} g_{s t, k}^{j}} \quad ; \quad 1 \geq k \geq K$,

where $K$ is the number of radial splines (i.e. 21) and $\mathbf{g}_{\mathrm{k}}^{\mathrm{j}}$ is a vector containing only the diagonal elements $g_{s t, k}^{j}$ of $\left(\mathbf{G}^{T} \mathbf{G}\right)_{k}^{j}$.

Fig. 5 shows the sensitivity of several subsets of the data to structure at each vertical spline. The surface-wave dispersion data constrain $\mathrm{d} \ln V_{S}$ in the upper mantle and mantle transition zone (splines 1-5) and $\operatorname{dn} V_{P}$ only in the uppermost mantle (splines 1 and 2). The traveltimes of the direct phases ( $S$ and $P$ ) are mainly sensitive to the lower mantle, whereas surface-reflected traveltimes (SS, SSS, PP, PPP) constrain the mid-mantle. The normal-mode splitting data constrain the mid- and lower mantle and they are especially well suited to constrain $\operatorname{d} \ln V_{P}$ in the lower mantle. The fundamental mode data also constrain $\operatorname{d} \ln V_{P}$ in the upper mantle and transition zone (splines 2-5). The CMB Stoneley mode data have optimal sensitivity to $\mathrm{d} \ln V_{S}$ and $\mathrm{d} \ln V_{P}$ in the $\mathrm{D}^{\prime \prime}$ region (i.e. splines 20 and 21).

The $\left(\mathbf{G}^{T} \mathbf{G}\right)$ matrix used to construct model SP12RTS is computed by a weighted sum of the individual $\left(\mathbf{G}^{T} \mathbf{G}\right)^{j}$ matrices for the three different data types $j$ :

$\left(\mathbf{G}^{T} \mathbf{G}\right)=\sum_{j=1}^{3} w_{j}\left(\mathbf{G}^{T} \mathbf{G}\right)^{j}$.

The weights $w_{j}$ are chosen to obtain uniform sensitivity across all radial splines and the lowest overall misfit (see Section 5). We choose relative weights of $w_{t}=20$ for traveltime and $w_{s}=1000$ for splitting function data (relative to dispersion data with $w_{d}=1$ ). These weights emphasise the contribution of normal-mode data, which provide the most uniform constraints on $\operatorname{d} \ln V_{S}$ and $\operatorname{d} \ln V_{P}$ in the lower mantle.

\section{LONG-WAVELENGTH MANTLE STRUCTURE ACCORDING TO MODEL SP12RTS}

Fig. 6 presents maps of $\operatorname{d} \ln V_{S}$ and $\operatorname{d} \ln V_{P}$ according to model SP12RTS at different depths in the mantle. SP12RTS shows the general characteristics observed in many global tomography models. Tectonics and continental-scale geology are dominant at $100 \mathrm{~km}$ depth, we observe slab flattening in the mantle transition zone and broad low-velocity structures become visible in the lower mantle. The largest velocity variations in SP12RTS are found in the mantle boundary layers (for $\operatorname{d} \ln V_{S}$ up to 3.5 per cent at the top boundary, the lithosphere and up to 1.5 per cent at the bottom boundary, the $\mathrm{D}^{\prime \prime}$ region). The amplitudes of $\mathrm{d} \ln V_{S}$ are higher than for $\mathrm{d} \ln V_{P}$, but their correlation is high as observed in previous joint long-wavelength $V_{S}$ and $V_{P}$ models (Su \& Dziewonski 1997; Ishii \& Tromp 1999; Masters et al. 2000; Simmons et al. 2010).

The $\mathrm{d} \ln V_{S}$ part of SP12RTS is not significantly different from S20RTS and S40RTS. The correlation between these pure $V_{S}$ models and $\operatorname{dn} V_{S}$ in SP12RTS is higher than $\sim 0.61$ for S20RTS and 0.75 for S40RTS, with a depth average of 0.82 and 0.88 , respectively. The correlation is higher if we only consider structure below $2000 \mathrm{~km}$ depth, with a depth average of 0.91 and 0.92 , respectively. Fig. 7 indicates that the spectral content of SP12RTS, S20RTS and S40RTS is similar, especially the dominance of degree two in the lower mantle. While Fig. 6 indicates that the amplitudes of $\operatorname{d} \ln V_{S}$ in SP12RTS are small relative to those reported for S40RTS, Fig. 7 illustrates that the power present up to degree $s=12$ is comparable. Since we have chosen to give strong weights to the normal-mode data, SP12RTS has more power in the even degrees than the odd degrees in the mid- and lower mantle compared to S20RTS and S40RTS.

In the remainder of this study, we focus our attention on the lower mantle, given our prime interest in the characteristics of the LLSVPs. 
(a) $\Re \mathbf{e}\left(\mathbf{R}_{21}\right)$ coefficient

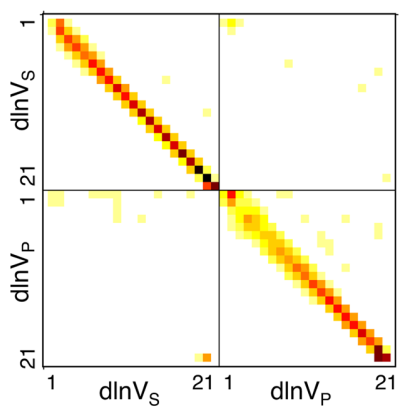

(d) $I m\left(R_{85}\right)$ coefficient

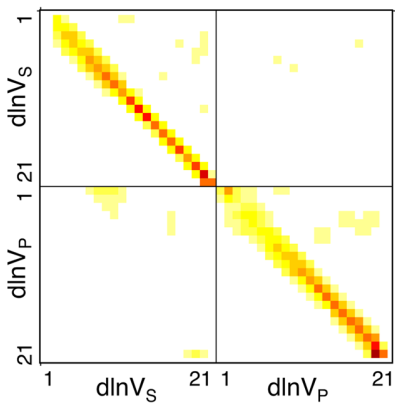

(b) $\Re e\left(R_{42}\right)$ coefficient

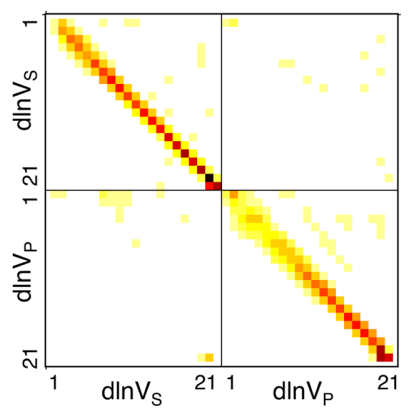

(e) $\Im m\left(R_{107}\right)$ coefficient

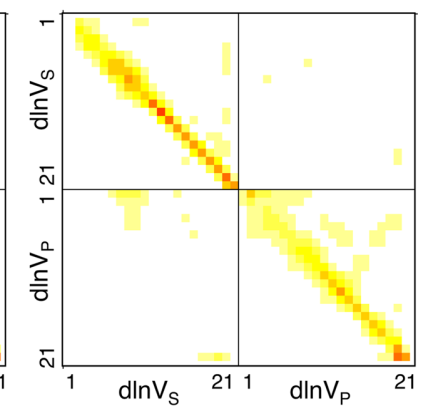

(c) $\Im m\left(R_{62}\right)$ coefficient

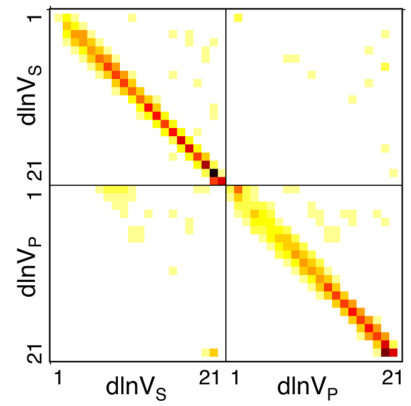

(f) $I m\left(R_{123}\right)$ coefficient

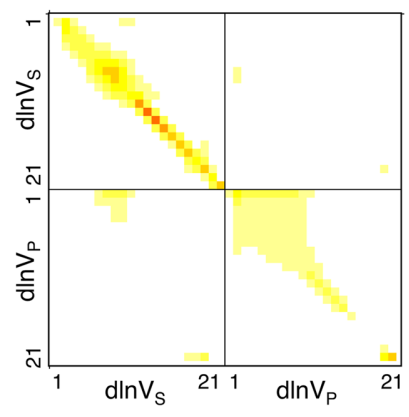

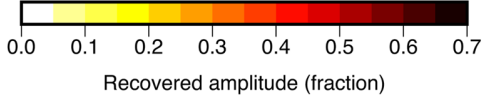

Figure 4. Graphical representation of coefficients $\mathcal{R}_{s t}$ of the resolution matrix as defined in eq. (8). The model coefficients are ordered such that the $V_{S}$ model coefficients are at the top left corner, those for $V_{P}$ in the bottom right corner and the other two blocks show the cross terms of the resolution matrix (e.g. projection of $V_{S}$ structure into $V_{P}$ and vice versa). In each block, there are contributions from the 21 radial spline functions which increase from the top to the bottom as indicated. We show examples for six coefficients of different spherical harmonic degree. Ideally, the resolution matrix should be the unit matrix. The resolution matrix presented here corresponds to that of the final SP12RTS model, which is presented in Section 4.

\subsection{Lower mantle characteristics}

Fig. 8 shows maps of $\operatorname{dn} V_{S}$ and $\operatorname{d} \ln V_{P}$ and histograms of the ratio $R=\mathrm{d} \ln V_{S} / \mathrm{d} \ln V_{P}$ computed by a point-by-point division. Large regions of low ( $S$ - and $P$-wave) velocity increase in strength towards the CMB. We will term these regions large-low-velocity provinces (LLVPs), because we confirm their existence in $V_{P}$ in addition to $V_{S}$. The LLVP under the Pacific is more round, whereas the LLVP under Africa is elongated in the north-south direction, as observed previously in $V_{S}$ models (Ritsema et al. 1999; McNamara \& Zhong 2005). While the amplitude of $\mathrm{d} \ln V_{S}$ increases continuously with depth, $V_{P}$ variations only increase significantly from $\sim 2600 \mathrm{~km}$ depth (Fig. 9a). This feature is reflected by $R$ in Fig. 8c; its median value is highest around $2500 \mathrm{~km}$ depth $(\sim 2.7)$ and $R$ decreases to a value of 1.4 near the CMB.

Fig. 9 shows radial depth profiles of the RMS velocity perturbations, the $\mathrm{d} \ln V_{S}-\mathrm{d} \ln V_{P}$ and $\mathrm{d} \ln V_{S}-\mathrm{d} \ln V_{C}$ correlations, and three estimates of the $\operatorname{dn} V_{S} / \operatorname{dn} V_{P}$ ratio $R$. A simple point-by-point division of $\operatorname{d} \ln V_{S}$ and $\operatorname{d} \ln V_{P}$ maps results in spurious estimates of $R$ in regions where $\operatorname{d} \ln V_{P}$ is close to zero. Therefore, we discard points where either $\left|\mathrm{d} \ln V_{S}\right|<0.1$ per cent or $\left|\mathrm{d} \ln V_{P}\right|<0.1$ per cent, similar to Della Mora et al. (2011), before computing the median values. We also estimate $R$ by simply dividing the RMS velocities, by using the model coefficients of (1) and (2), and from the slope of a regression fit between $\operatorname{d} \ln V_{S}$ and $\mathrm{d} \ln V_{P}$ (Ishii \& Tromp 2001). The estimates of $R$ obtained using the RMS velocities or the model coefficients are very similar, and we hence only show the former. The large RMS velocities in the uppermost and lowermost mantle, the high $\operatorname{d} \ln V_{S}-\mathrm{d} \ln V_{P}$ correlation at all depths, the negative $\mathrm{d} \ln V_{S}-\mathrm{d} \ln V_{C}$ correlation in the lower mantle from about $1500 \mathrm{~km}$ depth and the increase of $R$ with depth in the lower mantle up to $2500 \mathrm{~km}$ depth are characteristics of SP12RTS that are consistent with previous tomographic studies (e.g. Su \& Dziewonski 1997; Masters et al. 2000; Romanowicz 2001; Antolik et al. 2003; Houser et al. 2008) and traveltime data (e.g. Ritsema \& van Heijst 2002). Lower and upper bound estimates of $R$ are obtained from the median values and the regression fit values, respectively. All estimates suggest that $R$ decreases in the lowermost $300 \mathrm{~km}$ of the mantle (Fig. 9c), although the maximum value of $R$ around $2500 \mathrm{~km}$ depth varies from 2.7 to 5.4. In subsequent figures, we generally plot estimates of $R$ based on the RMS velocities, given these reflect intermediate values and are easily computed for previously published models.

The decrease of $R$ appears to be a robust feature of SP12RTS. Almost all previous studies (Fig. 10) agree that $R$ increases with depth in the lower mantle, although the absolute estimates of $R$ are highly variable (Figs $10 \mathrm{~b}$ and $\mathrm{c}$ ). Studies that show similarly high values as SP12RTS in the lowermost mantle are the normal-mode study of Romanowicz (2001) and the body-wave study of Su \& Dziewonski (1997). The pronounced decrease of $R$ in $\mathrm{D}^{\prime \prime}$ featured in SP12RTS is not a clear characteristic of other seismological models. The models by Antolik et al. (2003) and Della Mora et al. (2011) show a similar decrease of $R$, but they do not match SP12RTS at shallower depths. Saltzer et al. (2001) find a peak value of 2.7 at $2100 \mathrm{~km}$ depth, but lack resolution below a depth of $2500 \mathrm{~km}$. Romanowicz (2001), who also analysed normal-mode data, found a peak value of 
(a) $d \ln V_{S}$

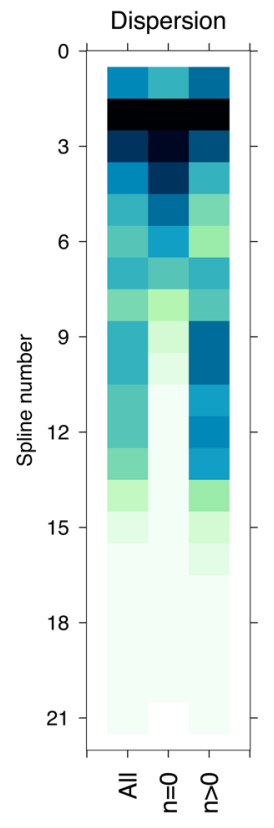

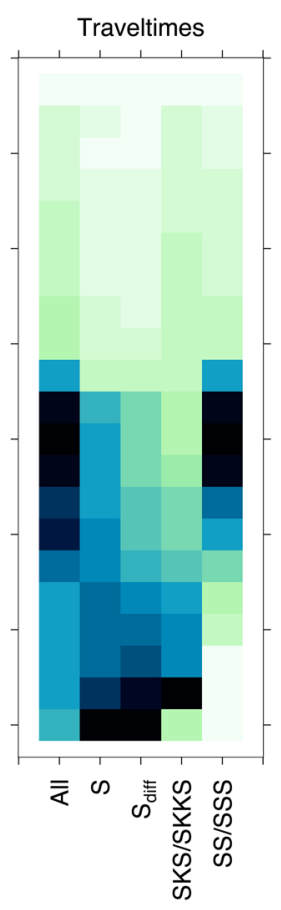

(b) dlnV

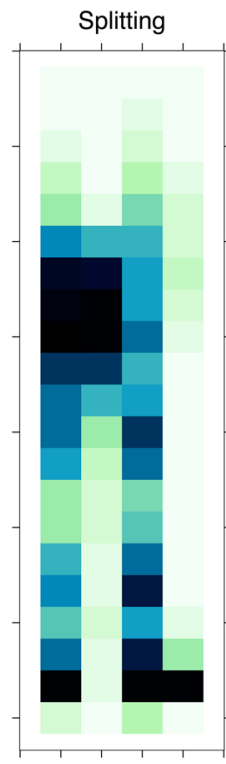

¿ II $\stackrel{0}{\underline{\Lambda}}$

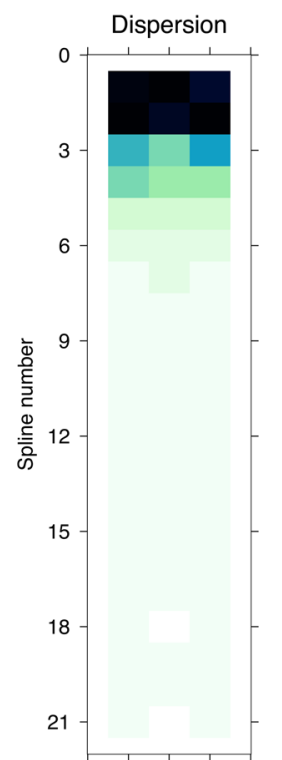

₹ 에 옫

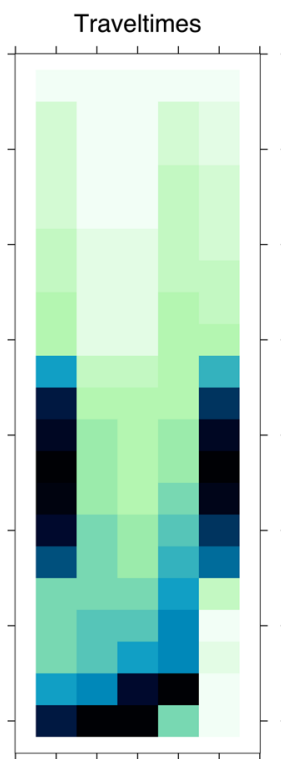

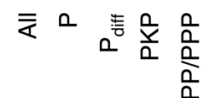

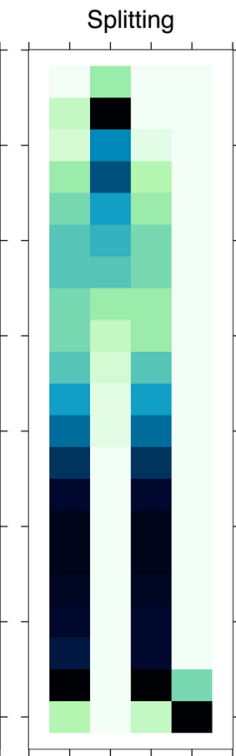

$\bar{\alpha}$

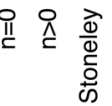

\section{EFFECT OF STONELEY MODES AND DATA WEIGHTING}

To verify whether the characteristics of SP12RTS are determined primarily by the Stoneley mode data, we compare SP12RTS with model SP12RTS_NST obtained without the nine Stoneley modes, that is, leaving out 349 coefficients from a total of 6970 coefficients. Like SP12RTS, SP12RTS_NST has 1200 resolved parameters to facilitate a quantitative comparison.

Fig. 11 shows maps of $\operatorname{d} \ln V_{S}$ and $\operatorname{dnn} V_{P}$ and corresponding histograms of $R$ for SP12RTS_NST. Both SP12RTS and SP12RTS_NST display the same large-scale patterns, but $\operatorname{dn} V_{S}$ at $2600 \mathrm{~km}$ depth and $\operatorname{dnn} V_{P}$ at $2850 \mathrm{~km}$ depth are stronger in SP12RTS. Figs 11(c) and 12 indicate that the decrease of $R$ in the $\mathrm{D}^{\prime \prime}$, and other SP12RTS characteristics are not determined by the Stoneley mode data alone. The decrease of $R$ is merely amplified

when Stoneley mode data are included. No significant changes occur in these curves above $\sim 2000 \mathrm{~km}$ depth, illustrating that the Stoneley mode data only constrain lowermost mantle structure.

Fig. 13 demonstrates that the depth profile of $R$ depends somewhat on the relative weights of the splitting and traveltime data. The versions of SP12RTS derived using relatively high weights for the splitting function data (models M_S_ALL and M_S_NST with $\left.w_{t}=1, w_{s}=1000\right)$ feature a stronger decrease of $R$ than models in which the traveltime data are weighted more strongly (models M_T_ALL and M_T_NST with $w_{t}=50, w_{s}=100$ ). This is primarily caused by higher shear-wave RMS velocities in M_T models than in M_S models, as reported previously (Gilbert \& Dziewonski 1975). This $S$-wave discrepancy and the higher values of $R$ found for M_T models could be due to finite-frequency effects on bodywave traveltimes (Nolet \& Moser 1993; Schuberth et al. 2012). We are currently investigating whether our ray-theoretical modelling of the traveltime data causes the differences in $R$ observed in Fig. 12. Nevertheless, we also find $R>2.5$ for models dominated by normal-mode data, which automatically include finite-frequency effects, thus requiring an additional explanation. The decrease of $R$ in $\mathrm{D}^{\prime \prime}$ is found irrespective of the different weights, and strongly preferred by the Stoneley mode data, though much less strong in traveltime dominated models M_T. However, we also observe a decrease of $R$ when the Stoneley mode data are excluded (models M_T_NST, SP12RTS_NST and M_S_NST).

Table 4 summarizes misfit values for different subsets of our data, calculated for the six versions of SP12RTS (Fig. 13). Generally, the 


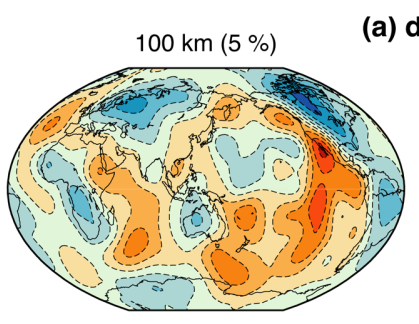

(a) $\operatorname{dln}_{\mathrm{s}}$
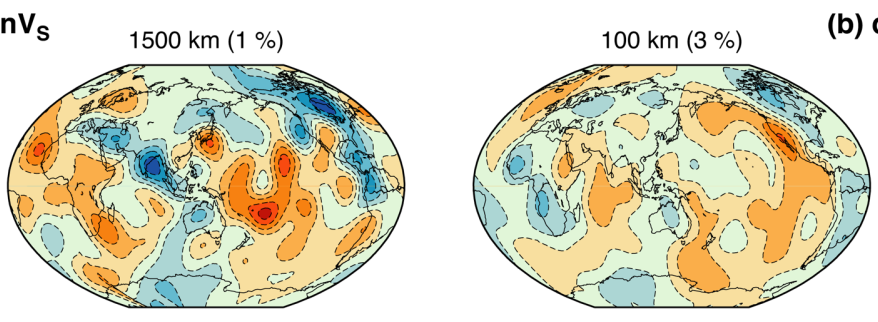

(b) $d \ln V_{p}$
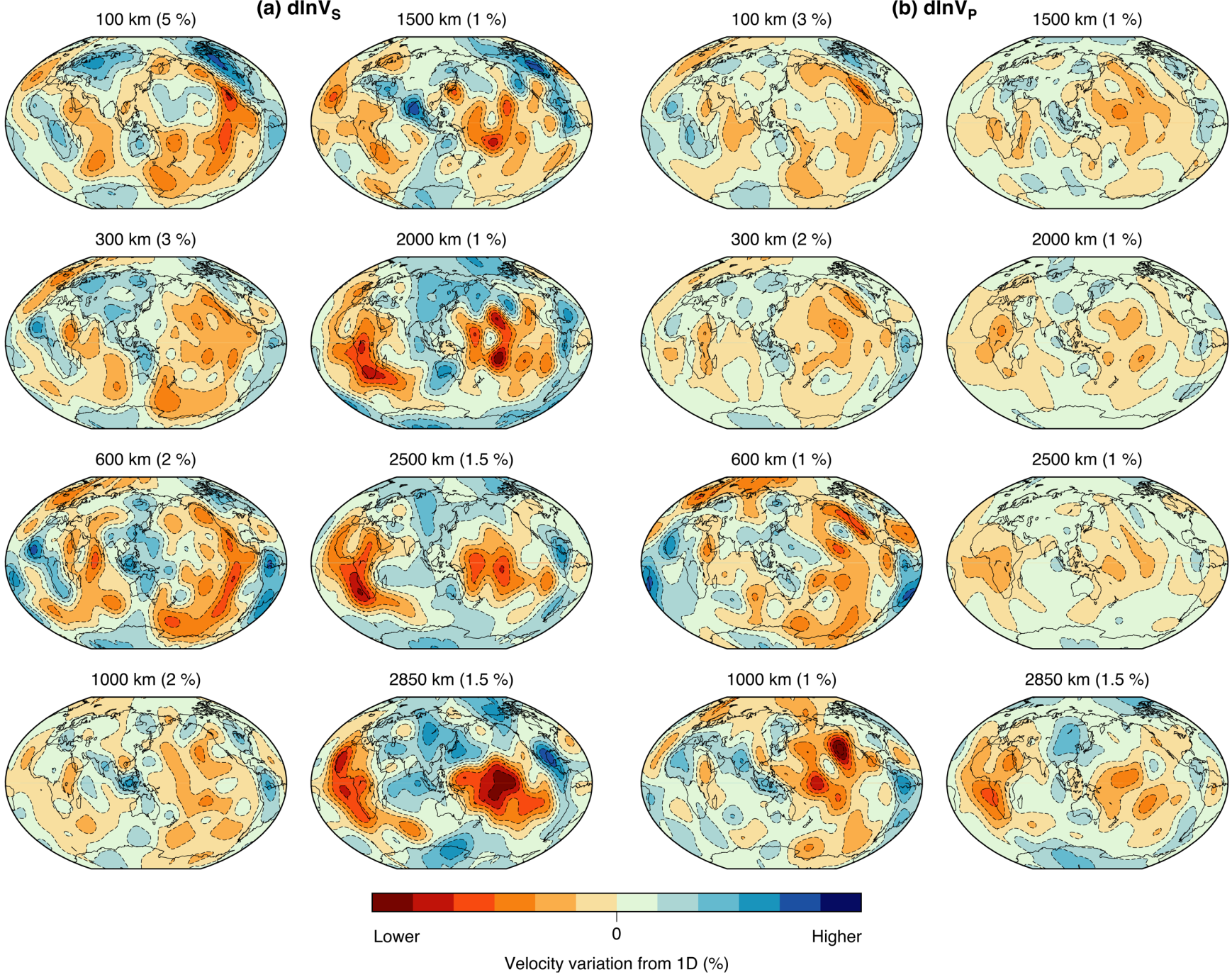

Figure 6. Maps of (a) shear-wave velocity variations $\operatorname{d} \ln V_{S}$ and (b) compressional-wave velocity variations $\operatorname{dln} V_{P}$ according to model SP12RTS at 100,300 , $600,1000,1500,2000,2500$ and $2850 \mathrm{~km}$ depth. Velocity is higher (lower) than the radially averaged value at each depth in blue (red) regions and the colour intensity is proportional to the amplitude of the variations up to the maximum (in per cent) indicated above each map.

(a) SP12RTS

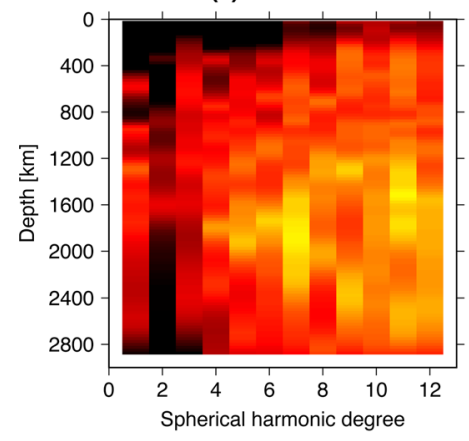

(b) S20RTS

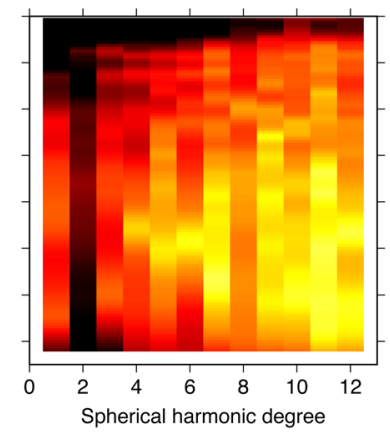

$\begin{array}{ll}-3.0 & -2.5\end{array}$ (c) S40RTS

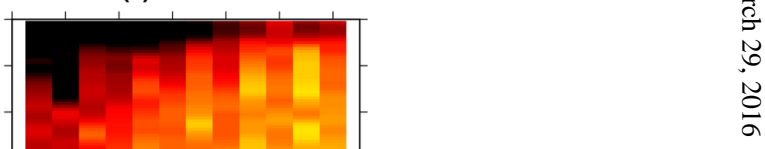

Figure 7. Spectral power of shear-wave velocity heterogeneity in tomographic models (a) SP12RTS, (b) S20RTS and (c) S40RTS. Spectral power is plotted up to $s=12$ on a logarithmic scale as a function of spherical harmonic degree and depth. 


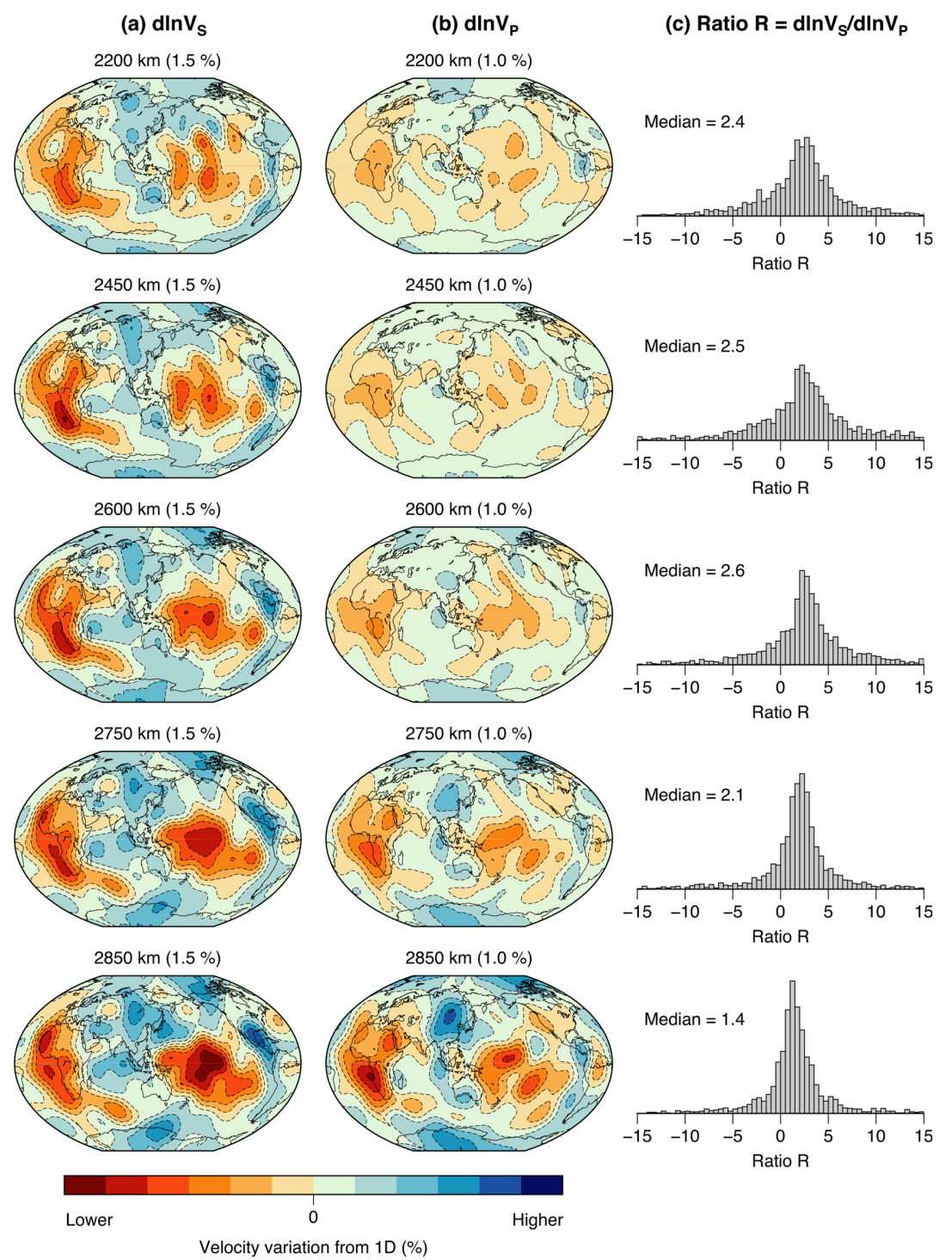

Figure 8. Maps of (a) shear-wave velocity variations $\operatorname{d} \ln V_{S}$ and (b) compressional-wave velocity variations dln $V_{P}$ according to model SP12RTS at depths of $2200,2450,2600,2750$ and $2850 \mathrm{~km}$ in the lowermost mantle. (c) Histograms of $R=\operatorname{d} \ln V_{S} / \operatorname{d} \ln V_{P}$ for these depths computed by taking a point-by-point ratio of all non-zero points at a given depth. At each depth, we indicate the median value of the histogram. See the caption of Fig. 6 for more details.

normal-mode data are fit better for models that incorporate Stoneley mode data (M_ALL models). As expected, the misfit reduction of Stoneley mode data is largest (17-36 per cent) when these data are included in the inversion. Additionally, all other mantle modes show small misfit reductions (0.6-3.5 per cent), indicating they also prefer model SP12RTS over SP12RTS_NST. In contrast, the bodywave data show negligible differences in misfit $(<0.6$ per cent $)$ between M_ALL and M_NST models. This is possibly because the total amount of heterogeneity in these models is similar, that is, the surface under the curves in Fig. 13 is more or less constant (compare model M_S_ALL and M_S_NST in Fig. 13a). The body-wave data may hence lack the depth resolution to distinguish between M_ALL and M_NST models. Consequently, the decrease of $R$ in $\mathrm{D}^{\prime \prime}$ is a feature strongly preferred by the normal-mode data. Whether it is compatible with the traveltimes of core-diffracted waves requires an analysis with realistic (finite-frequency) sensitivity kernels.

\section{CLUSTER ANALYSIS}

Several studies have estimated lateral variations of $R$ (e.g. Masters et al. 2000; Ishii \& Tromp 2001; Saltzer et al. 2001; Ritsema \& van Heijst 2002; Mosca et al. 2012). Some of these studies (Masters et al. 2000; Ishii \& Tromp 2001) suggested that $R$ is higher in the Pacific LLVP than in the SRs. Saltzer et al. (2001) indicated that $R$ is relatively high in areas not linked to subduction in the last $120 \mathrm{Myr}$ (e.g. outside the Pacific rim and the Alpine collision belt). Our histograms of $R$ (Fig. 8) also indicate that $R$ varies laterally at a given depth.

To estimate the lateral variations of $R$ in the lowermost mantle more quantitatively, we divide SP12RTS according to the cluster analysis of Lekić et al. (2012). Two clusters of relatively low and high velocities represent the LLVPs and the SRs, respectively. Fig. 14 shows that the RMS values of $\operatorname{d} \ln V_{S}$ and $\operatorname{d} \ln V_{P}$ are largest in the LLVPs. $\operatorname{dn} V_{S}$ and $\operatorname{dln} V_{P}$ in the LLVPs increase at shallower 
(a) RMS amplitude

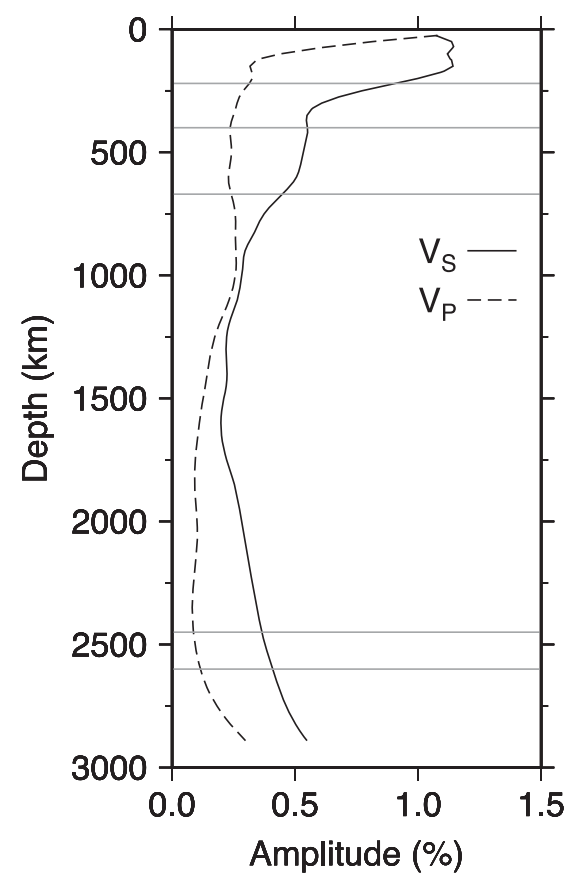

(b) Cross-correlation

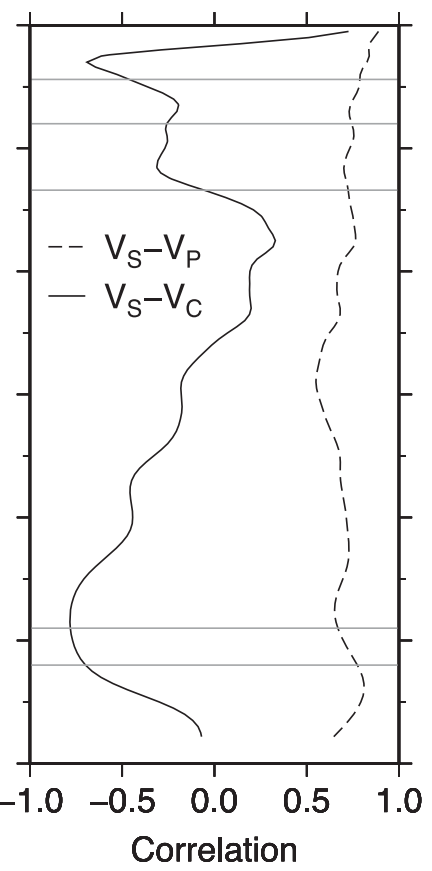

(c) $d \ln V_{S} / d \ln V_{P}$

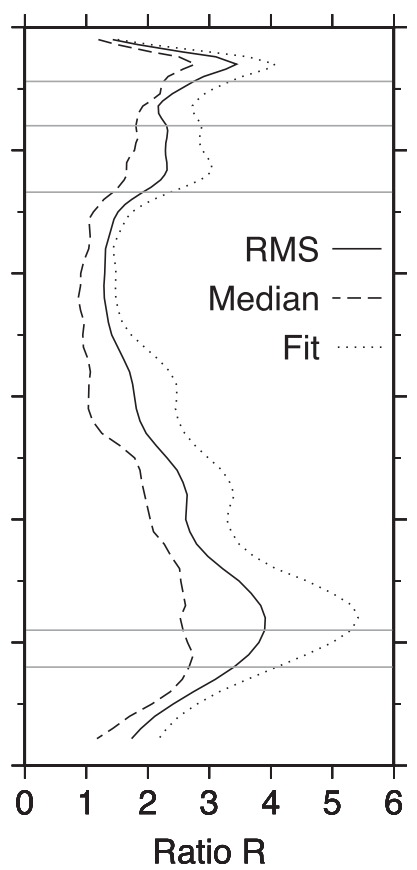

Figure 9. SP12RTS model characteristics showing (a) the RMS amplitudes of $V_{S}$ (solid) and $V_{P}$ (dashed) variations, (b) the dln $V_{S}$-dln $V_{P}$ (dashed) and $\mathrm{d} \ln V_{S}-\mathrm{d} \ln V_{C}$ (solid) correlation, and (c) the ratio $R=\mathrm{d} \ln V_{S} / \mathrm{d} \ln V_{P}$. The ratio in (c) is computed in three ways: (1) by dividing the RMS velocities (solid), (2) by taking the median of histograms derived by a point-by-point division of the corresponding maps (dashed) and (3) by the slope of a regression fit between $\mathrm{d} \ln V_{S}$ and $\operatorname{d} \ln V_{P}$ (dotted). Horizontal lines indicate the upper mantle discontinuities at 220, 410 and $660 \mathrm{~km}$ depth, as well as depths of 2450 and $2600 \mathrm{~km}$ depth for ease of reference.

(a) SP12RTS

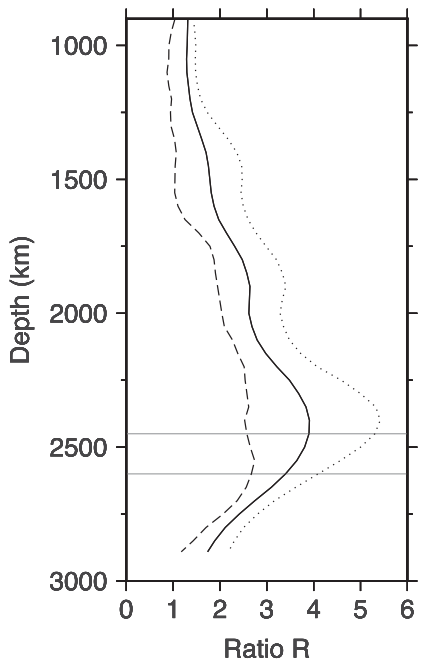

(b) Normal modes

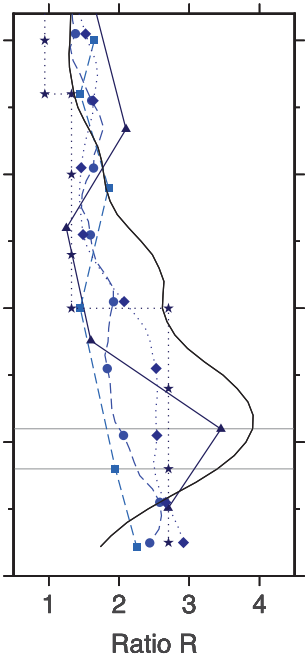

(c) Body waves

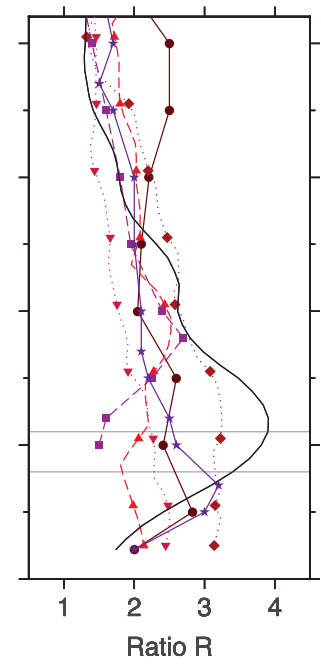

(d) Mineral physics

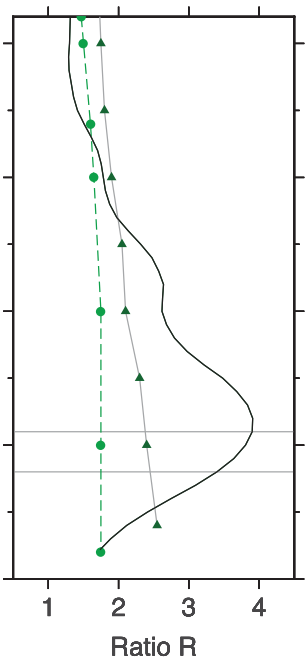

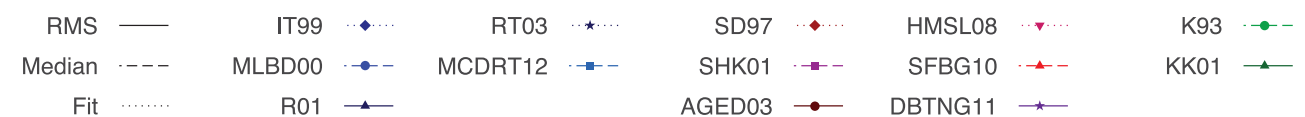

Figure 10. Depth profiles of $R$, showing values for (a) our model SP12RTS, (b) normal-mode data derived models, (c) models employing primarily body-wave data and (d) mineral physics predictions for purely thermal variations. For our model, we show the same three curves as in Fig. 9(c). Normal-mode models include IT99 (Ishii \& Tromp 1999), MLBD00 (Masters et al. 2000), R01 (Romanowicz 2001), RT03 (Resovsky \& Trampert 2003) and MCDRT12 (Mosca et al. 2012). Body-wave models include SD97 (Su \& Dziewonski 1997), SHK01 (Saltzer et al. 2001), AGED03 (Antolik et al. 2003 ), HMSL08 (Houser et al. 2008), SFBG10 (Simmons et al. 2010) and DBTNG11 (Della Mora et al. 2011). Mineral physics predictions are given for purely thermal variations for K93 (Karato 1993) and KK01 (Karato \& Karki 2001), where the latter includes the effect of anelasticity. Horizontal lines indicate depths of 2450 and 2600 km depth for ease of reference. 


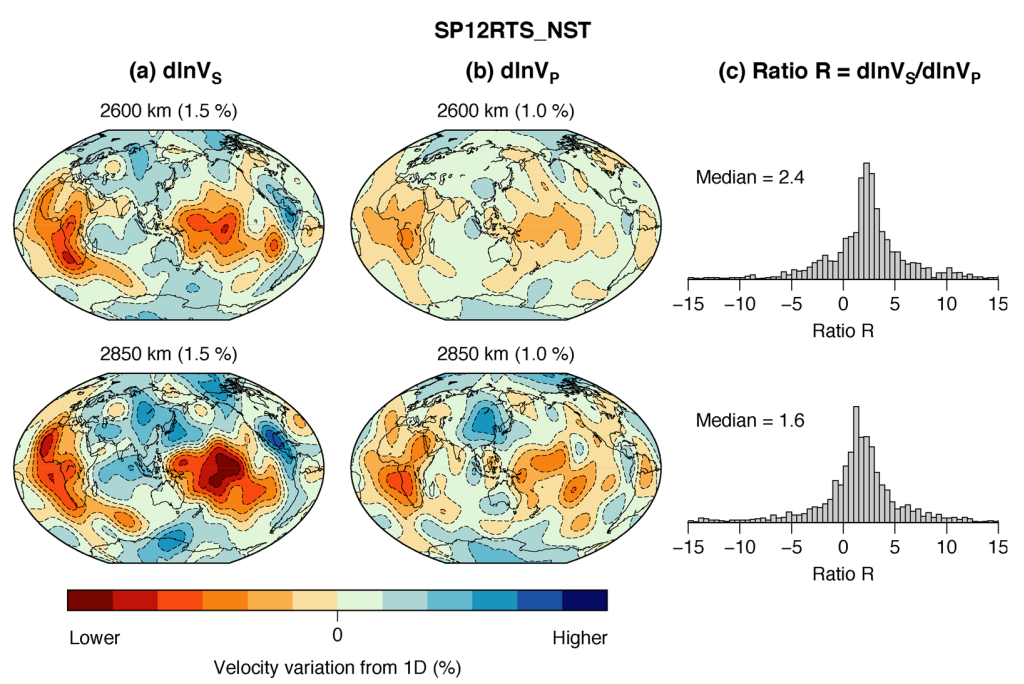

Figure 11. Model SP12RTS_NST without Stoneley mode data. We show maps at depths of 2600 and $2850 \mathrm{~km}$ for (a) shear-wave velocity variations dln $V_{S}$, (b) compressional-wave velocity variations $\operatorname{d} \ln V_{P}$ and (c) histograms of $R=\operatorname{d} \ln V_{S} / \operatorname{d} \ln V_{P}$, similar to Fig. 8 .

(a) $V_{S}$ RMS

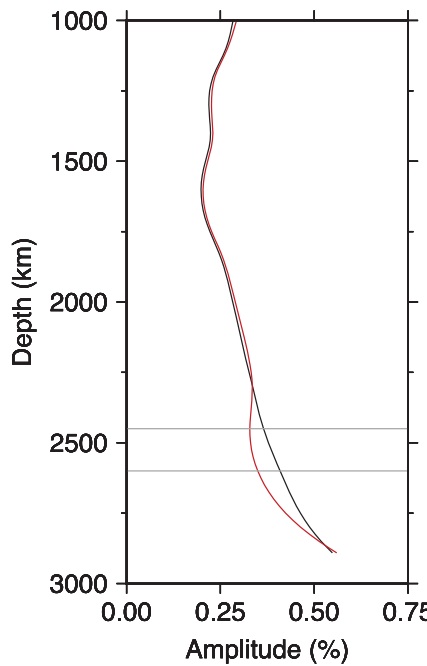

(b) $V_{p}$ RMS

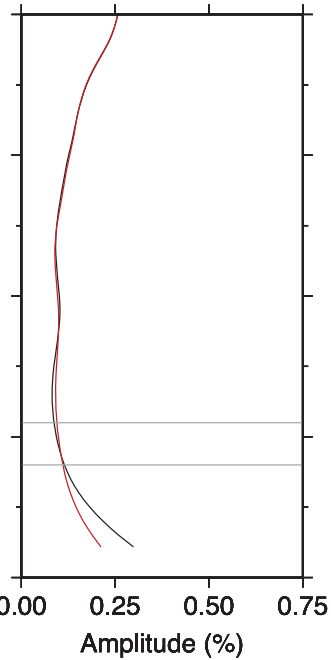

(c) $V_{s}-V_{c}$ correlation

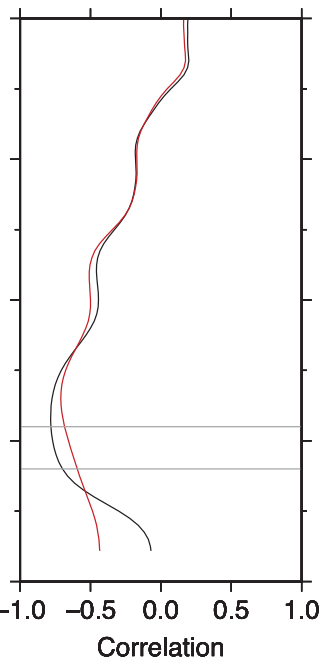

- SP12RTS depth, in agreement with Lekić et al. (2012). We also find $R$ to be higher in the LLVPs below a depth of $1500 \mathrm{~km}$, consistent with the work by Masters et al. (2000) and Ishii \& Tromp (2001). Both the LLVPs and the SRs show a negative correlation between $\operatorname{d} \ln V_{S}$ and $\mathrm{d} \ln V_{C}$ and a decrease of $R$ in $\mathrm{D}^{\prime \prime}$, with the peak occurring at similar depths for both clusters.

\section{DISCUSSION AND CONCLUDING REMARKS}

SP12RTS is a new 3-D model of $V_{S}$ and $V_{P}$ variations in the mantle derived from Rayleigh wave phase velocities, body-wave traveltimes and normal-mode splitting function measurements. The combination of $S$ - and $P$-wave traveltime measurements, $V_{P}$-sensitive normal-mode data and CMB Stoneley mode data provides optimal sensitivity to both shear-wave and compressional-wave velocity variations. SP12RTS shares many characteristics with previous tomographic models (e.g. Su \& Dziewonski 1997; Masters et al. 2000; Ishii \& Tromp 2001; Simmons et al. 2010; Mosca et al. 2012). It features a high correlation between the shear- and compressional-wave velocities, a negative correlation between the shear-wave and bulk-sound velocity below $1500 \mathrm{~km}$ depth and the ratio $R=\mathrm{d} \ln V_{S} / \mathrm{d} \ln V_{P}$ increases from $\sim 1.5$ at the top of the lower mantle to $\sim 4.0$ at $2500 \mathrm{~km}$ depth. It also contains large-lowvelocity-provinces (LLVPs) in the lowermost mantle for both $\mathrm{d} \ln V_{P}$ and $\operatorname{d} \ln V_{S}$.

In contrast to previous models, SP12RTS features a marked decrease of $R$ from a depth of $2500 \mathrm{~km}$ to the CMB. Previously, such a decrease has been attributed to bad data coverage. However, our 
(a) Splitting dominated

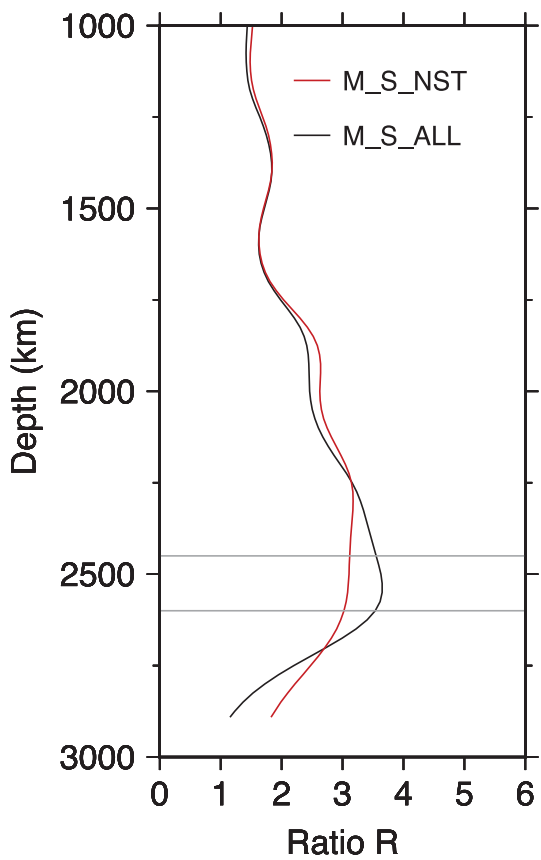

(b) Mixed

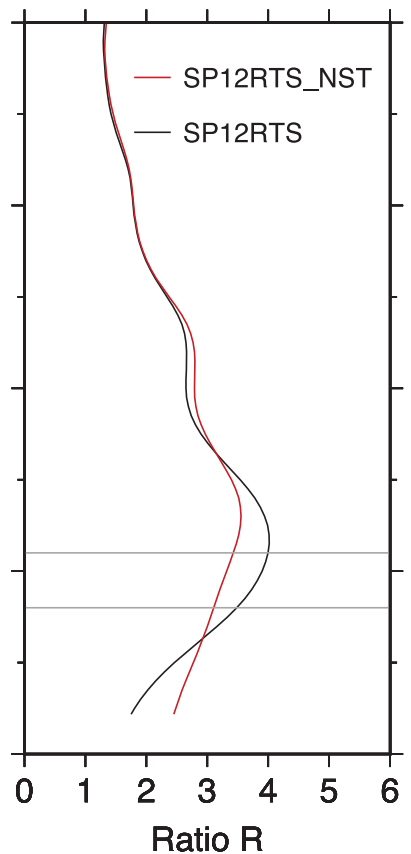

(c) Traveltimes dominated

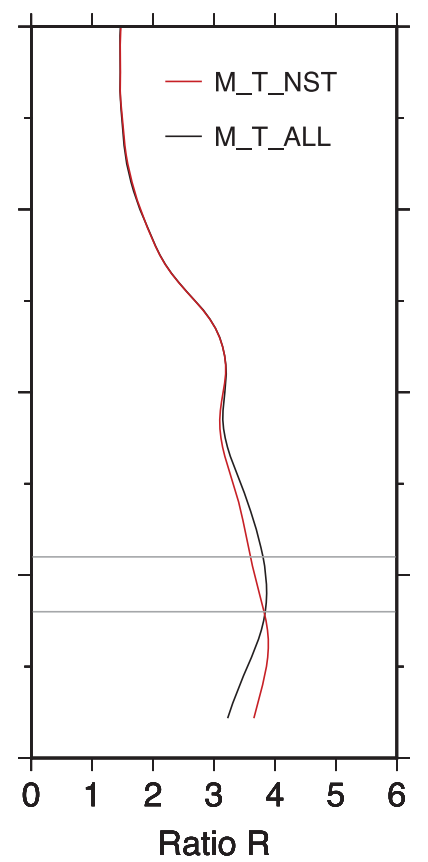

Figure 13. Depth profiles of $R$ computed using the RMS velocities, showing values for models with different weighting factors. We show (a) splitting data dominated models M_S_ALL and M_S_NST $\left(w_{t}=1\right.$ and $\left.w_{s}=1000\right)$, (b) mixed models SP12RTS and SP12RTS_NST $\left(w_{t}=20\right.$ and $\left.w_{s}=1000\right)$ and $(\mathrm{c})$ traveltime data dominated models M_T_ALL and M_T_NST $\left(w_{t}=50\right.$ and $\left.w_{s}=100\right)$. In each case, we show M_ALL models obtained using all data (black) and M_NST models that exclude Stoneley mode data (red).

Table 4. L2 misfit values for the six versions of SP12RTS with different relative traveltime $\left(w_{t}\right)$ and splitting $\left(w_{s}\right)$ data weights. We show misfit values for M_NST models (generated without Stoneley mode data) and for M_ALL models as well as the corresponding relative change in misfit. Averaged misfit values (normalized by the number of data in each case) are given for the $S$ - and $P$-wave traveltime data, the Stoneley modes, all modes and all other mantle modes excluding Stoneley modes. The M_ALL model with $w_{t}=20$ and $w_{s}=1000$ has on average the lowest misfit and this is therefore our preferred model SP12RTS. Bold values correspond to misfit values also reported in Tables 2 and 3 .

\begin{tabular}{llllr}
\hline Model & Data set & M_NST & M_ALL & $\begin{array}{r}\text { Relative change in misfit } \\
\text { (per cent) }\end{array}$ \\
\hline & Mantle modes & 0.1449 & 0.1399 & -3.41 \\
M_T & All modes & 0.1484 & 0.1400 & -5.67 \\
$w_{t}=50$ & Stoneley modes & 0.1696 & 0.1409 & -16.92 \\
$w_{s}=100$ & $P$-wave traveltimes & 0.4946 & 0.4951 & 0.08 \\
& S-wave traveltimes & 0.3156 & 0.3150 & -0.19 \\
& Mantle modes & 0.0911 & 0.0897 & -1.59 \\
SP12RTS & All modes & 0.0895 & $\mathbf{0 . 0 8 3 4}$ & -6.85 \\
$w_{t}=20$ & Stoneley modes & 0.0842 & 0.0566 & -32.78 \\
$w_{s}=1000$ & $P$-wave traveltimes & 0.5385 & $\mathbf{0 . 5 4 0 6}$ & 0.39 \\
& S-wave traveltimes & 0.3471 & $\mathbf{0 . 3 4 7 5}$ & 0.12 \\
& Mantle modes & 0.0812 & 0.0807 & -0.62 \\
M_S & All modes & 0.0795 & 0.0742 & -6.69 \\
$w_{t}=1$ & Stoneley modes & 0.0732 & 0.0469 & -35.97 \\
$w_{s}=1000$ & $P$-wave traveltimes & 0.6497 & 0.6534 & 0.57 \\
& $S$-wave traveltimes & 0.4390 & 0.4401 & 0.25 \\
\hline
\end{tabular}

resolution analysis demonstrates that the decrease of $R$ is a robust model feature, found independently of the damping value of the inversion and it provides a better fit to the mode data, in particular the Stoneley modes. A cluster analysis indicates that $R$ is highest in the LLVPs and that $R$ decreases near the CMB in both the LLVPS and the SRs.
High values of $R$ and the negative correlation between $\operatorname{d} \ln V_{S}$ and $\mathrm{d} \ln V_{C}$ point to the presence of chemical heterogeneity or $\mathrm{pPv}$ in the deep mantle. If these features are due to large-scale compositional variations in the lower mantle, the decrease of $R$ in the $\mathrm{D}^{\prime \prime}$ may indicate an increasing influence of the thermal boundary layer on seismic velocities (Saltzer et al. 2001). $R$ is also higher than expected 
(a) RMS amplitude

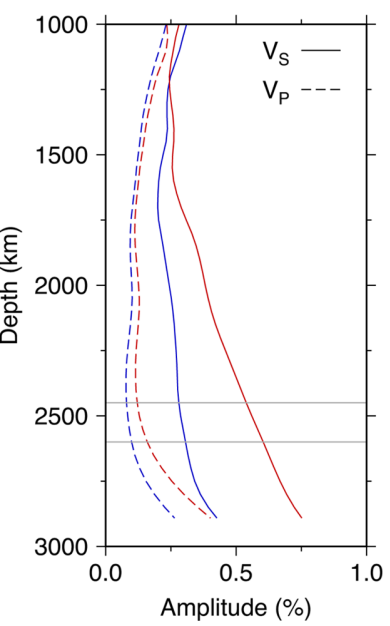

(b) $\mathrm{V}_{\mathrm{s}}-\mathrm{V}_{\mathrm{c}}$ correlation

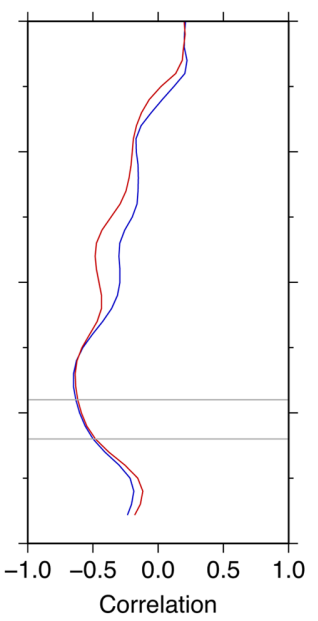

(c) $d \ln V_{S} / d \ln V_{P}$

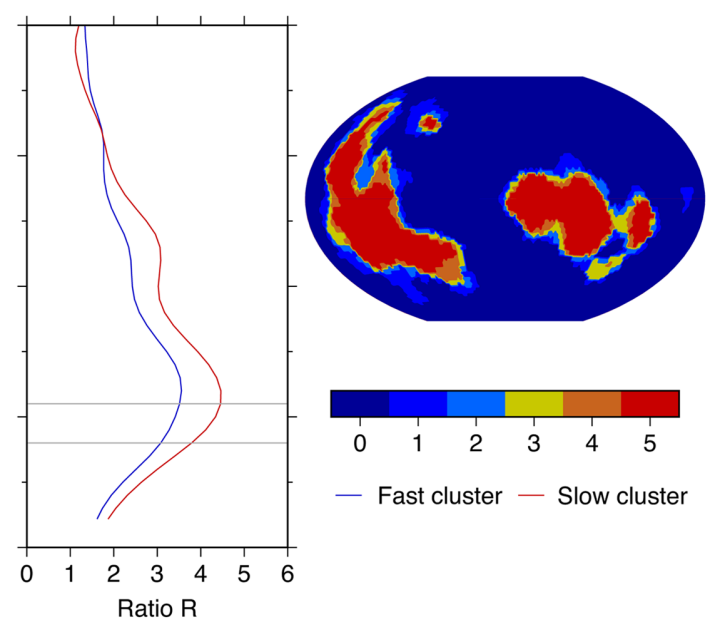

Figure 14. Depth profiles of SP12RTS showing (a) the RMS amplitudes of $V_{S}$ (solid) and $V_{P}$ (dashed) variations, (b) the dln $V_{S}$-dln $V_{C}$ correlation, (c) the ratio $R=\mathrm{d} \ln V_{S} / \mathrm{d} \ln V_{P}$ computed using the RMS velocities and (d) the lowermost mantle clusters of Lekic et al. (2012), which are used to compute the curves in (a)-(c). Yellow/red colours denote the slow cluster (LLVPs) and blue colours denote the fast cluster (SRs).

for purely thermal variations in the regions surrounding the LLVPs, implying that in this case compositional heterogeneity would be present in both clusters.

If $\mathrm{pPv}$ is solely responsible for the negative correlation between $\mathrm{d} \ln V_{S}$ and $\mathrm{d} \ln V_{C}$ in both clusters, the peak value of $R$ is expected at different depths in the LLVPs and SRs due to the Clapeyron slope of the transition. Such a depth offset is not observed in SP12RTS as the peak value of $R$ occurs at a depth of $\sim 2450 \mathrm{~km}$ in both the LLVPs and SRs (Fig. 14). The absence of this depth offset is likely due to vertical smearing of structure, which can be investigated by tomographically filtering the velocity structures obtained from geodynamic models. Alternatively, it may point to the presence of subducted MORB or iron in the slow cluster, which allows the stability field of pPv to extend higher above the CMB (Tateno et al. 2007; Ohta et al. 2008; Grocholski et al. 2012), hence not giving rise to a depth offset. Although it remains unclear whether $\mathrm{pPv}$ can be stable $>500 \mathrm{~km}$ above the $\mathrm{CMB}$, the effects of vertical smearing on the depth extent of the negative correlation between $\operatorname{dn} V_{S}$ and $\mathrm{d} \ln V_{C}$ should not be ignored.

To investigate the presence of large-scale chemical variations and $\mathrm{pPv}$ in the lowermost mantle, it is necessary to reconcile seismological observations with geodynamic models and mineral physics constraints. Similarly to RDHW11, tools to incorporate the characteristics of SP12RTS (also as a function of damping parameter) in such studies are made readily available.

\section{ACKNOWLEDGEMENTS}

This research was funded by the European Research Council (ERC) under the European Community's Seventh Framework Programme (FP7/2007-2013)/ERC grant agreement no. 204995. PK acknowledges funding from Pembroke College, Cambridge and an ETH Zürich Postdoctoral Fellowship (ETH/COFUND FEL-25 13-2, grant agreement no. 0-20997-14). AD is also funded by a Philip Leverhulme Prize and JR is supported by NSF grant EAR-1416695. We thank the Editor (Saskia Goes) and Lapo Boschi and Guust Nolet for constructive comments, which helped to improve the manuscript. We would like to thank Ved Lekic for providing his vote map of lower mantle clusters and we thank Ana Ferreira and Keith Priestley for helpful discussions. Figures have been produced using the GMT software (Wessel et al. 2013) and data were provided by IRIS/DMC.

\section{REFERENCES}

Antolik, M., Gu, Y.J., Ekström, G. \& Dziewonski, A.M., 2003. J362D28: a new joint model of compressional and shear velocity in the Earth's mantle, Geophys. J. Int., 153(2), 443-466.

Auer, L., Boschi, L., Becker, T., Nissen-Meyer, T. \& Giardini, D., 2014. Savani: a variable resolution whole-mantle model of anisotropic shear velocity variations based on multiple data sets, J. geophys. Res., 119(4), 3006-3034.

Bassin, C., Laske, G. \& Masters, G., 2000. The current limits of resolution for surface wave tomography in North America, EOS, Trans. Am. geophys. Un., 81, F897.

Boschi, L. \& Dziewonski, A.M., 2000. Whole Earth tomography from delay times of P, PcP, and PKP phases: lateral heterogeneities in the outer core or radial anisotropy in the mantle?, J. geophys. Res., 105(B6), $13675-$ 13696.

Bower, D.J., Gurnis, M. \& Seton, M., 2013. Lower mantle structure from paleogeographically constrained dynamic Earth models, Geochem. Geophys. Geosyst., 14(1), 44-63.

Chang, S.-J., Ferreira, A.M.G., Ritsema, J., van Heijst, H.J. \& Woodhouse, J.H., 2015. Joint inversion for global isotropic and radially anisotropic mantle structure including crustal thickness perturbations, J. geophys. Res., 120, 4278-4300.

Cobden, L., Thomas, C. \& Trampert, J., 2015. Seismic detection of postperovskite inside the Earth, in The Earth's Heterogeneous Mantle eds Deschamps, F. \& Khan, A., pp. 391-440, Springer International Publishing.

Davaille, A., 1999. Simultaneous generation of hotspots and superswells by convection in a heterogeneous planetary mantle, Nature, 402(6763), 756-760.

Davies, D. \& Davies, J., 2009. Thermally-driven mantle plumes reconcile multiple hot-spot observations, Earth planet. Sci. Lett., 278(1), 50-54.

Davies, D., Goes, S., Davies, J., Schuberth, B., Bunge, H. \& Ritsema, J., 2012. Reconciling dynamic and seismic models of Earth's lower mantle: the dominant role of thermal heterogeneity, Earth planet. Sci. Lett., 353, 253-269. 
Della Mora, S., Boschi, L., Tackley, P.J., Nakagawa, T. \& Giardini, D., 2011. Low seismic resolution cannot explain S/P decorrelation in the lower mantle, Geophys. Res. Lett., 38(12), L12303, doi:10.1029/2011GL047559.

Deschamps, F. \& Tackley, P.J., 2009. Searching for models of thermochemical convection that explain probabilistic tomography: II. Influence of physical and compositional parameters, Phys. Earth planet. Inter., 176(1), 1-18.

Deschamps, F., Trampert, J. \& Tackley, P.J., 2007. Thermo-chemical structure of the lower mantle: seismological evidence and consequences for geodynamics, in Superplume: Beyond Plate Tectonics, pp. 293-320, Springer Publishing Group.

Deuss, A., Ritsema, J. \& van Heijst, H.-J., 2013. A new catalogue of normalmode splitting function measurements up to $10 \mathrm{mHz}$, Geophys. J. Int., 192(3), 920-937.

Dziewonski, A. \& Anderson, D., 1981. Preliminary reference Earth model, Phys. Earth planet. Inter., 25(4), 297-356.

Dziewonski, A.M., Hager, B.H. \& O'Connell, R.J., 1977. Large-scale heterogeneities in the lower mantle, J. geophys. Res., 82(2), 239-255.

Dziewonski, A.M., Lekić, V. \& Romanowicz, B.A., 2010. Mantle anchor structure: an argument for bottom up tectonics, Earth planet. Sci. Lett., 299(1), 69-79.

Edmonds, A., 1960. Angular Momentum in Quantum Mechanics, Princeton Univ. Press.

Ferreira, A.M.G., Woodhouse, J.H., Visser, K. \& Trampert, J., 2010. On the robustness of global radially anisotropic surface wave tomography, J. geophys. Res., 115(B4), B04313, doi:10.1029/2009JB006716.

French, S., Lekić, V. \& Romanowicz, B., 2013. Waveform tomography reveals channeled flow at the base of the oceanic asthenosphere, Science, 342(6155), 227-230.

Garnero, E. \& McNamara, A., 2008. Structure and dynamics of Earth's lower mantle, Science, 320(5876), 626-628.

Gilbert, F. \& Dziewonski, A., 1975. An application of normal mode theory to the retrieval of structural parameters and source mechanisms from seismic spectra, Phil. Trans. R. Soc. Lond., 278(1280), 187-269.

Grocholski, B., Catalli, K., Shim, S.-H. \& Prakapenka, V., 2012. Mineralogical effects on the detectability of the postperovskite boundary, Proc. Natl. Acad. Sci. USA, 109(7), 2275-2279.

Gu, Y.J., Dziewonski, A.M., Su, W. \& Ekström, G., 2001. Models of the mantle shear velocity and discontinuities in the pattern of lateral heterogeneities, J. geophys. Res., 106(B6), 11 169-11 199.

Houser, C., Masters, G., Shearer, P. \& Laske, G., 2008. Shear and compressional velocity models of the mantle from cluster analysis of long-period waveforms, Geophys. J. Int., 174(1), 195-212.

Iitaka, T., Hirose, K., Kawamura, K. \& Murakami, M., 2004. The elasticity of the $\mathrm{MgSiO}_{3}$ post-perovskite phase in the Earth's lowermost mantle, Nature, 430(6998), 442-445.

Ishii, M. \& Tromp, J., 1999. Normal-mode and free-air gravity constraints on lateral variations in velocity and density of Earth's mantle, Science, 285(5431), 1231-1236.

Ishii, M. \& Tromp, J., 2001. Even-degree lateral variations in the Earth's mantle constrained by free oscillations and the free-air gravity anomaly, Geophys. J. Int., 145(1), 77-96.

Karato, S., 1993. Importance of anelasticity in the interpretation of seismic tomography, Geophys. Res. Lett., 20(15), 1623-1626.

Karato, S.-i. \& Karki, B.B., 2001. Origin of lateral variation of seismic wave velocities and density in the deep mantle, J. geophys. Res., 106(R10), $21771-21783$.

Koelemeijer, P.J., 2014. Normal mode studies of long wavelength structures in Earth's lowermost mantle, $P h D$ thesis, pp. 1-254, University of Cambridge.

Koelemeijer, P., Deuss, A. \& Ritsema, J., 2013. Observations of coremantle boundary Stoneley modes, Geophys. Res. Lett., 40(11), 25572561.

Koelemeijer, P., Deuss, A. \& Ritsema, J., 2015. Earth's hot lowermost mantle revealed by Stoneley mode splitting observations, Nature Comm., in press.

Kustowski, B., Ekström, G. \& Dziewoński, A.M., 2008. Anisotropic shearwave velocity structure of the Earth's mantle: a global model, J. geophys. Res., 113(B6), B06306, doi:10.1029/2007JB005169.
Lay, T. \& Garnero, E.J., 2011. Deep mantle seismic modeling and imaging, Ann. Rev. Earth planet. Sci., 39, 91-123.

Lay, T., Hernlund, J. \& Buffett, B., 2008. Core-mantle boundary heat flow, Nature Geosci., 1(1), 25-32.

Lekić, V., Cottaar, S., Dziewonski, A. \& Romanowicz, B., 2012. Cluster analysis of global lower mantle tomography: a new class of structure and implications for chemical heterogeneity, Earth planet. Sci. Lett., 357, 68-77.

Li, C., van der Hilst, R.D., Engdahl, E.R. \& Burdick, S., 2008. A new global model for P wave speed variations in Earth's mantle, Geochem. Geophys. Geosyst., 9(5), Q05018, doi:10.1029/2007GC001806.

Li, X., Giardini, D. \& Woodhouse, J., 1991a. The relative amplitudes of mantle heterogeneity in $\mathrm{P}$ velocity, S velocity and density from freeoscillation data, Geophys. J. Int., 105(3), 649-657.

Li, X., Giardini, D. \& Woodhouses, J., 1991b. Large-scale three-dimensional even-degree structure of the Earth from splitting of long-period normal modes, J. geophys. Res., 96(B1), 551-577.

Malcolm, A.E. \& Trampert, J., 2011. Tomographic errors from wave front healing: more than just a fast bias, Geophys. J. Int., 185(1), 385-402.

Masters, G., Laske, G., Bolton, H. \& Dziewonski, A., 2000. The relative behavior of shear velocity, bulk sound speed, and compressional velocity in the mantle: implications for chemical and thermal structure, $A m$. geophys. Un. Monogr., 117, 63-87.

McNamara, A., Garnero, E. \& Rost, S., 2010. Tracking deep-mantle reservoirs with ultra-low-velocity zones, Earth planet. Sci. Lett., 299(1), 1-9.

McNamara, A.K. \& Zhong, S., 2005. Thermochemical structures beneath Africa and the Pacific Ocean, Nature, 437(7062), 1136-1139.

Menke, W., 1989. Geophysical data analysis: discrete inverse theory, in Geophysical Data Analysis, pp. 1-289, Academic, San Diego, California.

Mosca, I., Cobden, L., Deuss, A., Ritsema, J. \& Trampert, J., 2012. Seismic and mineralogical structures of the lower mantle from probabilistic tomography, J. geophys. Res., 117(B6), B06304, doi:10.1029/2011JB008851.

Murakami, M., Hirose, K., Kawamura, K., Sata, N. \& Ohishi, Y., 2004. Postperovskite phase transition in $\mathrm{MgSiO}_{3}$, Science, 304(5672), 855-858.

Nakagawa, T. \& Tackley, P.J., 2014. Influence of combined primordial layering and recycled MORB on the coupled thermal evolution of Earth's mantle and core, Geochem. Geophys. Geosyst., 15(3), 619-633.

Nakagawa, T., Tackley, P.J., Deschamps, F. \& Connolly, J.A.D., 2012. Radial 1-D seismic structures in the deep mantle in mantle convection simulations with self-consistently calculated mineralogy, Geochem. Geophys. Geosyst., 13(11), Q11002, doi:10.1029/2012GC004325.

Nolet, G. \& Moser, T.-J., 1993. Teleseismic delay times in a 3-D earth and a new look at the s discrepancy, Geophys. J. Int., 114(1), 185-195.

Oganov, A. \& Ono, S., 2004. Theoretical and experimental evidence for a post-perovskite phase of $\mathrm{MgSiO}_{3}$ in Earth's D" layer, Nature, 430(6998), 445-448.

Ohta, K., Hirose, K., Lay, T., Sata, N. \& Ohishi, Y., 2008. Phase transitions in pyrolite and MORB at lowermost mantle conditions: implications for a MORB-rich pile above the core-mantle boundary, Earth planet. Sci. Lett., 267(1), 107-117.

Panning, M. \& Romanowicz, B., 2006. A three-dimensional radially anisotropic model of shear velocity in the whole mantle, Geophys. J. Int., 167(1), 361-379.

Panning, M., Lekić, V. \& Romanowicz, B., 2010. Importance of crustal corrections in the development of a new global model of radial anisotropy, J. geophys. Res., 115(B12), B12325, doi:10.1029/2010JB007520.

Resovsky, J. \& Ritzwoller, M., 1999. Regularization uncertainty in density models estimated from normal mode data, Geophys. Res. Lett., 26(15), 2319-2322.

Resovsky, J. \& Trampert, J., 2003. Using probabilistic seismic tomography to test mantle velocity-density relationships, Earth planet. Sci. Lett., 215(12), 121-134.

Ritsema, J. \& van Heijst, H.-J., 2002. Constraints on the correlation of P-and S-wave velocity heterogeneity in the mantle from P, PP, PPP and PKPab traveltimes, Geophys. J. Int., 149(2), 482-489.

Ritsema, J., van Heijst, H.-J. \& Woodhouse, J., 1999. Complex shear wave velocity structure imaged beneath Africa and Iceland, Science, 286(5446), 1925-1928. 
Ritsema, J., van Heijst, H.-J. \& Woodhouse, J.H., 2004. Global transition zone tomography, J. geophys. Res., 109(B2), B02302, doi:10.1029/2003JB002610

Ritsema, J., Deuss, A., van Heijst, H.-J. \& Woodhouse, J.H., 2011. S40RTS: a degree-40 shear-velocity model for the mantle from new Rayleigh wave dispersion, teleseismic traveltime and normal-mode splitting function measurements, Geophys. J. Int., 184(3), 1223-1236.

Romanowicz, B., 2001. Can we resolve 3D density heterogeneity in the lower mantle?, Geophys. Res. Lett., 28(6), 1107-1110.

Saltzer, R., van der Hilst, R. \& Karason, H., 2001. Comparing P and S wave heterogeneity in the mantle, Geophys. Res. Lett., 28(7), 1335-1338.

Schuberth, B.S.A., Bunge, H.-P., Steinle-Neumann, G., Moder, C. \& Oeser, J., 2009. Thermal versus elastic heterogeneity in high-resolution mantle circulation models with pyrolite composition: high plume excess temperatures in the lowermost mantle, Geochem. Geophys. Geosyst., 10(1), Q01W01, doi:10.1029/2008GC002235.

Schuberth, B.S.A., Zaroli, C. \& Nolet, G., 2012. Synthetic seismograms for a synthetic Earth: long-period P- and S-wave traveltime variations can be explained by temperature alone, Geophys. J. Int., 188(3), 1393-1412.

Simmons, N.A., Forte, A.M., Boschi, L. \& Grand, S.P., 2010. GyPSuM: a joint tomographic model of mantle density and seismic wave speeds, J. geophys. Res., 115(B12), B12310, doi:10.1029/2010JB007631.

Soldati, G., Boschi, L. \& Forte, A., 2012. Tomography of coremantle boundary and lowermost mantle coupled by geodynamics, Geophys. J. Int., 189(2), 730-746.

Su, W. \& Dziewonski, A., 1997. Simultaneous inversion for 3-D variations in shear and bulk velocity in the mantle, Phys. Earth planet. Inter., 100(1-4), $135-156$.
Tarantola, A., 1987. Inverse Problem Theory, Elsevier.

Tateno, S., Hirose, K., Sata, N. \& Ohishi, Y., 2007. Solubility of FeO in $(\mathrm{Mg}, \mathrm{Fe}) \mathrm{SiO}_{3}$ perovskite and the post-perovskite phase transition, Phys. Earth planet. Inter., 160(3), 319-325.

Trampert, J., Deschamps, F., Resovsky, J. \& Yuen, D., 2004. Probabilistic tomography maps chemical heterogeneities throughout the lower mantle, Science, 306(5697), 853-856.

Tsuchiya, T., Tsuchiya, J., Umemoto, K. \& Wentzcovitch, R., 2004. Phase transition in $\mathrm{MgSiO}_{3}$ perovskite in the Earth's lower mantle, Earth planet. Sci. Lett., 224(3-4), 241-248.

van Heijst, H.-J. \& Woodhouse, J., 1997. Measuring surface-wave overtone phase velocities using a mode-branch stripping technique, Geophys. J. Int., 131(2), 209-230.

van Heijst, H.-J. \& Woodhouse, J., 1999. Global high-resolution phase velocity distributions of overtone and fundamental-mode surface waves determined by mode branch stripping, Geophys. J. Int., 137(3), 601620

Wessel, P., Smith, W.H.F., Scharroo, R., Luis, J. \& Wobbe, F., 2013. Generic Mapping Tools: improved version released, EOS, Trans. Am. geophys. Un., 94(45), 409.

Woodhouse, J., Giardini, D. \& Li, X., 1986. Evidence for inner core anisotropy from free oscillations, Geophys. Res. Lett., 13(13), 1549-1552.

Wookey, J., Stackhouse, S., Kendall, J.-M., Brodholt, J. \& Price, G.D., 2005. Efficacy of the post-perovskite phase as an explanation for lowermost-mantle seismic properties, Nature, 438(7070), 1004 1007.

Young, M., Tkalcić, H., Bodin, T. \& Sambridge, M., 2013. Global P wave tomography of Earth's lowermost mantle from partition modeling, $J$. geophys. Res., 118(10), 5467-5486. 